\title{
MASA CR71.283
}

NATIONAL AERONAUTICS AND SPACE ADMINISTRATION

Technical Report No. 32-868

\section{Rigid Motions in Einstein Spaces}

\author{
Hugo D. Wahlquist
}

Frank B. Estabrook

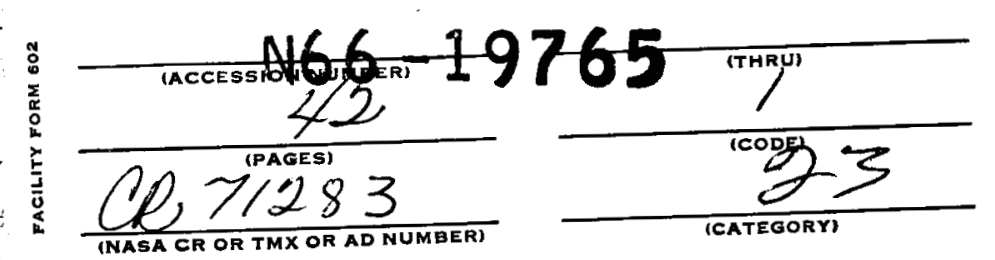

\section{GPO PRICE \$ CFSTI PRICE(S)}

Hard copy $(H C)$

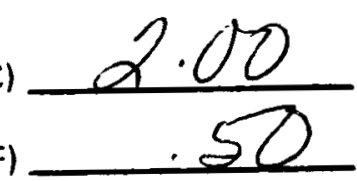

Microfiche (MF)

H 653 July 65

JET PROPULSION LABORATORY
CALIFORNIA INSTITUTE OF TECHNOLOGY

Pasadena, California

March 15, 1966 
NATIONAL AERONAUTICS AND SPACE ADMINISTRATION

Technical Report No. $32-868$

\section{Rigid Motions in Einstein Spaces}

Hugo D. Wahlquist

Frank B. Estabrook

R. J. Mackin, Jr., Manager
Physics Section

JET PROPULSION LABORATORY

CALIFORNIA INSTITUTE OF TECHNOLOGY

PASADENA, CALIFORNIA

March 15, 1966 
Copyright (C) 1966

Jet Propulsion Laboratory

California Institute of Technology

Prepared Under Contract No. NAS 7-100

National Aeronautics \& Space Administration 


\section{CONTENTS}

I. Introduction . . . . . . . . . . I

II. Dyadic Formalism and Equations . . . . . . . . . . . . 3

A. Intrinsic Reference Systems . . . . . . . . . . . . . . . 3

B. Co-Moving or Adapted Coordinates . . . . . . . . . . . . . . 4

C. Differentiation of Scalars, Vectors, and Dyadics . . . . . . . . . . 6

D. The Dyadic Equations for Rigid Reference Frames in Einstein Space . . . . . . . . . . . . . . . . 8

III. Rigid Motion in Space-Time of Constant Curvature. . . . . . 11

IV. Geodesic Rigid Frames . . . . . . . . . . . . . . . . . . . . . 15

V. Irrotational Rigid Frames .

V. Rotating Rigid Frames . . . . . . . . . . . . . . . . . . . . . . . . . 24

Appendix A. Summary of Dyadic Notation and Identities . . . . 28

Appendix B. Summary of the General Dyadic Equations . . . . . . 30

References . . . . . . . . . . . . . . . . . . . . . . . . . 34

\section{FIGURE}

1. Moving polar coordinates based on a curve $\mathcal{C}$. . . . . . . . . . . . . 24 


\begin{abstract}$$
19765
$$

The dyadic formulation of general relativity is used systematically to discuss rigid congruences in Einstein space-time. For space-time of uniform curvature, the quotient space metrics of rotating and accelerating rigid bodies are obtained. For Einstein space-time of nonuniform curvature, all irrotational, nonisometric, rigid motions are explicitly displayed. They have one degree of freedom, and occur only in degenerate static metrics of Class B. Rotating rigid congruences in Einstein space-time of nonuniform curvature are shown to have no degrees of freedom. Their evolution is, in fact, found to be governed by a complete set of 14 first-order total differential equations, linear in the time derivatives of the dyadic variables. Such rotating motions are shown further to be constrained by a set of algebraic conditions; the implication of this for the validity of the Herglotz-Noether theorem in Einstein space-time is discussed.
\end{abstract}<smiles>[Mg][Mg]</smiles>

\title{
I. INTRODUCTION
}

In 1908, Max Born (Ref. 1) proposed a Lorentzcovariant kinematic definition of rigidity in special relativity based on constancy of proper distance between adjacent material points. His definition seems appropriate for a rigid body, as it is mathematically equivalent to the vanishing of the rate-of-strain tensor. Born's constraint may also be geometrically characterized by the statement that the metric of the local 3-space (quotient space), orthogonal to the world lines of the rigid body, is constant through time.

It was soon demonstrated independently by $\mathrm{G}$. Herglotz and F. Noether (Refs. 2 and 3) that, in the Minkowski space-time of special relativity, this constraint leads to the surprising result that a rigid body, so defined, has in general only three degrees of freedom. Almost all allowed rigid motions are nonrotating. The very few special motions with rotation have the property that both the absolute acceleration and angular velocity vectors at each point of the body are constant in time relative to the body. This latter class of motions is, in fact, identical with the ten-parameter family of isometries of Minkowski space-time (transformations which carry the entire fourdimensional manifold into itself). This result, stating that the only allowed rigid motions with rotation are isometries of the space-time manifold, is today often referred to as the Herglotz-Noether theorem. 
Their proof of the theorem for flat space-time led to the conclusion that the ordinary physical concept of rigidity cannot be successfully incorporated in special relativity. Little further work on the subject appeared until much later when its possible importance for the general theory of relativity emerged as in, for example, the remarks of Synge (Ref. 4). The constraint of vanishing rate of strain may be carried over directly into general relativity (Refs. 5 and 6 ). Such rigid motions in curved space-time are of considerable interest: (1) mathematically for their possible use in the invariant geometric analysis of the structure and global properties of Riemannian manifolds, analogous to that based on the more restricted isometries; and (2) physically for attaining a better understanding of the imprecise concept of rigidity so often implicitly involved in the interpretation of relativistic experiments, e.g., the Pound-Rebka red-shift experiment.

The investigation of possible Born rigid motions in general relativity divides rather naturally into two different categories:

(1) The simplest is the study of test rigid bodies, which have zero (or at least negligible) stress-energy densities and so do not themselves contribute to the space-time curvature.

(2) The study of the dynamical rigid body having a nonneglectable stress-energy tensor.

A further important distinction must be made according to whether the space occupied by the rigid body contains stress-energy arising from other physical entities, in addition to any associated with the rigid body itself. It is usual to adopt the attitude that situations having superimposed, foreign, stress-energy through which the rigid body moves are of lesser physical interest, and so exclude them. The problem of test rigid bodies is accordingly restricted to empty or, if the cosmological constant is present, Einstein spaces. In the dynamical case, one would be led to require only that the velocity 4-vector of the rigid body be the timelike eigenvector of the Einstein or stress-energy tensor. This condition would ensure the vanishing of the local, proper momentum-density vector (denoted $\mathbf{t}$ in dyadic notation) inside the rigid body; it is also, of course, satisfied a fortiori in Einstein space, with or without cosmological constant.

Some of the questions which may be posed for study are:

(1) Does either the test or dynamical rigid body have more than the three degrees of freedom permitted in flat space-time?
(2) Is the time behavior of acceleration and angular velocity vectors restricted, and if so, how?

(3) Will an arbitrarily given Einstein space admit any test rigid body motion?

(4) Will such a space (empty, but perhaps with cosmological constant) admit rotating rigid motions other than isometries; i.e., does the HerglotzNoether theorem still apply?

In 1959, C. B. Rayner (Ref. 7) showed that the proper energy density and the magnitude of the angular velocity of a rigid body must be constant in time. Recently, Pirani and Williams (Ref. 8) have included this in a set of six relations between the time behavior of the angular velocity vector of the body and certain components of the space-time curvature; these relations follow from the time constancy of the orthogonal metric of the rigid body. R. H. Boyer (Ref. 9) has carefully discussed the entire problem and has extended this work to obtain sufficient, but not necessary, conditions for the validity of the Herglotz-Noether theorem in curved manifolds.

In this report, the equations of test rigid motion in Einstein space are systematically discussed. We use the dyadic notation and formalism for general relativity presented in Ref. 10. Indeed a collateral purpose of this report is to supplement the previous discussion at several important points, and then, above all, to demonstrate how the rather elegant and straightforward manipulations allowed by the dyadic equations can lead to useful results which otherwise, in covariant terminology, appear to be of impenetrable complexity.

In Section II, we introduce first the use of intrinsically determined reference vector frames, a device allowed by the dyadic formalism, which can be as important and convenient as the use of intrinsic coordinates. The use of co-moving intrinsic coordinates is discussed, as well as the various operations of differentiation. Finally, the basic dyadic equations are presented for test rigid frames in Einstein space.

In Section III, partly as an illustration of dyadic techniques, we prove the Herglotz-Noether theorem in space-time of uniform curvature (de Sitter space), a proof already far from trivial in covariant language. We derive, in this space, the general quotient-space (threedimensional, or inner) metrics of rotating and accelerating 
test rigid bodies, and briefly discuss the nonrotating (or normal) rigid motions (those which allow three degrees of freedom).

In Section IV, we prove a theorem stated by Boyer (Ref. 9) that geodesic test rigid frames are impossible in Einstein space (other than flat). In Section V, we show that nonrotating, nonisometric rigid motions can only occur in a restricted class of Type D Einstein spaces, and that such motions are allowed but one degree of freedom. The metrics and the rigid frames are exhibited. These are the only rigid motions that have any degree of freedom whatever in Einstein spaces which are neither flat nor uniformly curved.
In Section VI, we explicitly attack the HerglotzNoether theorem in Einstein space. The six timederivative equations of Pirani and Williams (Ref. 8) are shown to be included in a full set of 14 , which may be, in principle, solved for the local time rate of change of the 14 dyadic components involved, subject to a set of subsidiary algebraic conditions. A rigid frame can rotate only in the unlikely event that the subsidiary conditions are all fulfilled throughout the motion; the equations then determine the complete evolution of the motion and the metric from any initial 3-space. It remains to show that the subsidiary conditions can be satisfied only by isometries, if the Herglotz-Noether theorem is to be stated for general Einstein spaces in the usual simple fashion.

\section{DYADIC FORMALISM AND EQUATIONS}

\section{A. Intrinsic Reference Sysfems}

The dyadic formalism results from the introduction of an orthonormal tetrad of basis vectors, one timelike and three spacelike, at each event in space-time. The orthonormality relations may be written

$$
\begin{aligned}
& \mathbf{u}^{0} \cdot \mathbf{u}^{0}=-1, \\
& \mathbf{u}^{0} \cdot \mathbf{u}^{a}=0, \quad \mathbf{u}^{a} \cdot \mathbf{u}^{b}=\delta^{a b}, \quad a, b=1,2,3 .
\end{aligned}
$$

The timelike tetrad vector $\mathbf{u}^{0}$ is everywhere aligned with a given timelike congruence and so coincides with the unit tangent to the congruence. If the world lines of the congruence represent the history of a material medium, $\mathbf{u}^{0}$ will be the 4-velocity field of the matter. The spacelike triad $\mathbf{u}^{a}$ [or $\mathbf{u}, \mathbf{v}, \mathbf{w}$ ] then provides a locally co-moving frame of reference, and spans the local 3-space orthogonal to the given congruence at each event. A formal partitioning of the space-time manifold is thus achieved and by projecting all tensor fields of interest into these orthogonal 3 -spaces, we arrive at the three-dimensional vector and dyadic formalism. Some features of the dyadic notation are reviewed in Appendix A. The development of the general dyadic equations describing an arbitrary timelike congruence is sketched in Appendix B. A complete discussion of this approach is contained in Ref. 10.

In general, the local orthogonal 3-spaces at different events will not mesh together to form a family of immersed three-dimensional sub-spaces in the space-time manifold, nor is it generally the case that they are so 
related as to determine a metric on the quotient 3-space defined by the congruence. Further, the relation between the spacelike triads at different points is arbitrary, though assumed continuous, so that the tetrad field is, in general, anholonomic or nonintegrable. That is to say, the orthogonal basis vectors underlying the dyadic formulation are not necessarily related to any holonomic coordinate system in either three or four dimensions, nor derivable from scalars by differentiation.

The freedom within the dyadic formalism to use anholonomic reference vectors is often very convenient. It permits us to choose reference vectors intrinsically at each point of the manifold in such a way that the algebraic structure of the pertinent quantities and equations is as simple as possible. Typically, we might select an orthonormal triad formed from the acceleration and angular velocity vectors of the timelike congruence; again, the three eigenvectors of a field dyadic might be convenient. Reference vectors so chosen, however, may well be anholonomic. Ultimately, the comparison of conditions at different points of the manifold requires the introduction of a coordinate system and investigation of differential properties. The point is that such questions are easily deferred and studied as a completely separate issue after the algebraic characteristics of the problem are settled.

It is frequently the case that a given problem further provides its own intrinsic coordinates, as well as intrinsic reference vectors. This is a familiar situation in general relativity where the general covariance of the equations permits the election of almost any scalar variable to serve as a coordinate, at least over a limited region. As often suggested, the scalar invariants of the curvature tensor itself may be adopted. The dyadic formalism is most appropriate when a physically or geometrically distinguished timelike congruence exists in the space-time manifold, and this additional structure usually generates further scalar magnitudes from which intrinsic coordinates may be selected. The most convenient intrinsic coordinates arise from this last class, and are the comoving coordinates next discussed. When a coordinate system is finally adopted, the so-called natural system of reference vectors belonging to it may be introduced. In contrast to the original orthonormal tetrads, these new vectors will be derived from the coordinates by differentiation, and are consequently a holonomic intrinsic reference system. In the following two sub-sections, the relations between these different basis systems are developed.

\section{B. Co-Moving or Adapted Coordinates}

The most useful coordinate systems for our purposes are the adapted or co-moving coordinates. These are specified by taking the given timelike congruence curves to be the coordinate lines: $t$ varies, $x^{\alpha}$ constant $(\alpha=1,2,3)$. Each world line of the congruence is thus the intersection of three 3-spaces given by a particular set of constant values for the $x^{\alpha}$. Using the dot notation for the derivative with respect to proper time along the congruence, we clearly have $\dot{x}^{\alpha}=0$; in effect, the adapted coordinates $x^{\alpha}$ label the world lines. At each point, a natural holonomic triad of basis vectors $\mathbf{e}^{\alpha}$ is defined relative to a particular co-moving coordinate system by the normals to the three hypersurfaces, $x^{\alpha}=$ constant, passing through that point. Note that, by the definition of co-moving coordinates, these normal vectors will be everywhere orthogonal to the timelike congruence (which lies in the hypersurfaces $x^{\alpha}=$ constant), and so span the same local orthogonal 3 -space as the orthonormal triad $\mathbf{u}^{a}$. Using the normal gradient vector, we may define

$$
\mathbf{e}^{\alpha} \equiv \nabla x^{\alpha}
$$

where the $\nabla$ operator always denotes three-dimensional covariant differentiation orthogonal to the timelike congruence. Note that the index $\alpha$ on boldface symbols such as $\mathrm{e}^{\alpha}$ labels vectors, not components of a vector. A further dual set of natural vectors $\mathbf{e}_{\alpha}$ may be derived from $\mathbf{e}^{\alpha}$ by the inversion

$$
\mathbf{e}_{\alpha} \cdot \mathbf{e}^{\beta}=\delta_{\alpha}^{\beta} .
$$

As is familiar, these dual vectors will lie along the intersections of the coordinate surfaces. The dual of the orthonormal triad coincides with itself: $\mathbf{u}^{a}=\mathbf{u}_{a}$.

An arbitrary vector, say $\mathbf{V}$, orthogonal to the timelike congruence now has three possible expansions. Using the summation convention for repeated indices (from 1 to 3 , for either Greek or Latin indices from the first parts of their respective alphabets), we can write

$$
\begin{aligned}
\mathbf{V} & =\left(\mathbf{V} \cdot \mathbf{u}^{a}\right) \mathbf{u}_{a}=V^{a} \mathbf{u}_{a}, \\
& =\left(\mathbf{V} \cdot \mathbf{e}^{\alpha}\right) \mathbf{e}_{\alpha}=V^{a} \mathbf{e}_{\alpha}, \\
& =\left(\mathbf{V} \cdot \mathbf{e}_{\alpha}\right) \mathbf{e}^{\alpha}=V_{\alpha} \mathbf{e}^{\alpha},
\end{aligned}
$$

where $V^{a}=V_{a}$ are the orthonormal components, while $V^{\alpha}$ are contravariant, and $V_{\alpha}$ covariant, natural components. The dyadic I defined by

$$
\mathbf{I}=\mathbf{u}^{a} \mathbf{u}_{a}=\mathbf{e}^{\alpha} \mathbf{e}_{\alpha}=\mathbf{e}_{\alpha} \mathbf{e}^{\alpha}
$$


serves as the unit operator;

$$
\mathbf{V} \cdot \mathbf{I}=\mathbf{I} \cdot \mathbf{V}=\mathbf{V} .
$$

We may also expand these sets of basis vectors on each other so that

$$
\left.\begin{array}{l}
\mathbf{e}^{\alpha}={ }_{a} \lambda^{\alpha} \mathbf{u}^{a}, \\
\mathbf{e}_{\alpha}={ }^{a} \lambda_{\alpha} \mathbf{u}_{a}, \\
\mathbf{u}^{a}=\mathbf{u}_{a}={ }^{a} \lambda_{\alpha} \mathbf{e}^{a}={ }_{a} \lambda^{\alpha} \mathbf{e}_{\alpha},
\end{array}\right\}
$$

thus defining the connecting quantities, or transformation matrices, ${ }_{a} \lambda^{\alpha},{ }^{a} \lambda_{\alpha}$. These will satisfy the equations

$$
{ }_{a} \lambda^{\alpha a}{ }^{a} \lambda_{\beta}=\delta_{\beta}^{\alpha}, \quad{ }_{a} \lambda^{\alpha b} \lambda_{\alpha}=\delta_{a}^{b},
$$

which result directly from Eqs. (1) and (3). The ${ }_{a} \lambda^{\alpha}$ are simply the ordinary coordinate tensor notation for the triad vectors $\mathbf{u}_{a}$. The dyadic formalism was developed in this language in Ref. 10 and is also employed in Appendix B of this report.

The dual sets of natural vectors are useful for expressing the metric of the local orthogonal 3-space in terms of an adapted coordinate system. Taking various natural components of the unit dyadic, we have

$$
\mathbf{e}^{\alpha} \cdot \mathbf{i} \cdot \mathbf{e}_{\beta}=\mathbf{e}^{\alpha} \cdot \mathbf{e}_{\beta}=\hat{\delta}_{\beta}^{\tilde{\beta}},
$$

and define

$$
\begin{aligned}
& h_{\alpha \beta} \equiv \mathbf{e}_{\alpha} \cdot 1 \cdot \mathbf{e}_{\beta}=\mathbf{e}_{\alpha} \cdot \mathbf{e}_{\beta}, \\
& h^{\alpha \beta} \equiv \mathbf{e}^{\alpha} \cdot 1 \cdot \mathbf{e}^{\beta}=\mathbf{e}^{\alpha} \cdot \mathbf{e}^{\beta} .
\end{aligned}
$$

Thus, for instance, a displacement vector $\mathbf{d x}$, orthogonal to the timelike congruence, will have the natural expansion

$$
\mathbf{d x}=\left(\mathbf{d x} \cdot \mathbf{e}^{\alpha}\right) \mathbf{e}_{\alpha}=d x^{\alpha} \mathbf{e}_{\alpha},
$$

and the norm

$$
d x^{2}=\mathbf{d x} \cdot \mathbf{d x}=\mathbf{e}_{\alpha} \cdot \mathbf{e}_{\beta} d x^{\alpha} d x^{\beta}=h_{\alpha \beta} d x^{\alpha} d x^{\beta}
$$

Finally, we introduce at each event the natural vector $\mathbf{e}^{0}$, normal to the coordinate surface $t=$ constant through that event, and write its expansion in the orthonormal tetrad. There is no requirement that the $t$-coordinate surfaces be everywhere orthogonal to the timelike congru- ence (for rotating matter this will not even be possible), so that the normal to these surfaces will have projections both along, and orthogonal to, the congruence. Thus, we write

$$
\mathbf{e}^{0}=\left(\mathbf{e}^{0} \cdot \mathbf{u}_{10}\right) \mathbf{u}^{0}+\left(\mathbf{e}^{0} \cdot \mathbf{u}_{a}\right) \mathbf{u}^{a},
$$

and it is convenient to introduce the special symbols

$$
\phi \equiv \mathbf{e}^{0} \cdot \mathbf{u}_{n,}, \quad \mathbf{A} \equiv\left(\mathbf{e}^{0} \cdot \mathbf{u}_{a}\right) \mathbf{u}^{a},
$$

so that

$$
\mathbf{e}^{0}=\boldsymbol{\phi} \mathbf{u}^{0}+\mathbf{A} .
$$

The quantity $\phi$ is a scalar, while $\mathbf{A}$ is a vector in the orthogonal 3-space; they represent the absolute rate of change of the $t$-coordinate, respectively along, and orthogonal to, the timelike congruence, and could as well be defined by

$$
\phi=\frac{d t}{d \tau}=\dot{t}, \quad \mathbf{A}=\nabla t
$$

where $\tau$ is proper time on the timelike congruence. The quantities $\phi$ and $A$ serve in the dyadic formulation as potentials for the inertial field described by the acceleration a and angular velocity $\Omega$ of the timelike congruence. [By grace of the principle of equivalence, the local field, determined by the orthonormal reference system and "absolutely" measurable with accelerometers and gyroscopes gimballed in that system (the field $-a$ and $-\boldsymbol{\Omega}$ ), is often called the gravitational field. The formal analogy of test particle behavior with that of charges in an electromagnetic field $\mathbf{E}$ and $\mathbf{B}$ is indeed close. But it is clear that better terminology is to denote $a$ and $\Omega$ as the inertial field, reserving the gravitation epithet for the small perturbations of the inertial field expressed by the ten components of the Weyl dyadics A and B (Ref. 10).] As verified in Appendix $B$, the potential equations take the form

$$
\phi \mathbf{a}=-\nabla_{\phi}+\dot{\mathbf{A}}+\mathbf{S}^{\star} \cdot \mathbf{A}
$$

and

$$
2 \phi \Omega=\nabla \times A,
$$

closely analogous to the potential equations of electromagnetism. The existence of such potentials is guaranteed by the possibility of using co-moving coordinates, and through their identification with derivatives of the $t$-coordinate as in Eq. (16). 
These potentials are not unique; "gauge transformations" are allowed similar to those of electromagnetism, and it is easily verified with the use of the commutation relations [Eqs. (B-20) and (B-21) of Appendix B] that the new potentials

$$
\bar{\phi}=\phi+\dot{\psi}, \quad \overline{\mathbf{A}}=\mathbf{A}+\nabla \psi,
$$

with $\psi$ an arbitrary scalar function, reproduce Eqs. (17) and (18). Recalling the definitions of the original $\phi$ and A in Eq. (16), it is clear that we are simply introducing a new time coordinate, $\bar{t}=t+\psi$, where

$$
\bar{\phi}=\dot{\bar{t}}, \quad \overline{\mathbf{A}}=\nabla \bar{t} .
$$

The special case, $\bar{t}=f(t)$ with $f(t)$ an arbitrary function, is often useful since the potentials are transformed only by a factor,

$$
\bar{\phi}=\left(\frac{d f}{d t}\right)_{\phi}, \quad \overline{\mathbf{A}}=\left(\frac{d f}{d t}\right) \mathbf{A} .
$$

If we are dealing with an irrotational congruence $(\Omega=0$ ), it will be simplest to use a "Coulomb" gauge such that $\mathbf{A}=\mathbf{0}$. The transformation of Eq. (21) then permits multiplication of $\phi$ by an arbitrary function of time without destroying this condition on $\mathrm{A}$.

Using Eq. (15), the contravariant components of the four-dimensional space-time metric can be expressed as

$$
\begin{aligned}
& g^{\prime \mu \prime}=\mathbf{e}^{\alpha} \cdot \mathbf{e}^{0}=\mathbf{A}^{z}-\phi^{2}, \quad g^{0 \alpha}=\mathbf{e}^{n} \cdot \mathbf{e}^{\alpha}=\mathbf{A} \cdot \mathbf{e}^{\alpha}=A^{\alpha}, \\
& g^{\alpha \beta}=\mathbf{e}^{\alpha} \cdot \mathbf{e}^{\beta}=h^{\alpha \beta} .
\end{aligned}
$$

The inversion, $g_{\mu v} g^{v \tau}=\delta_{\mu}^{\tau}$, determines the covariant components to be

$$
\begin{aligned}
& g_{m+1}=-\frac{1}{\phi^{2}}, \quad g_{\alpha \mu}=\frac{1}{\phi^{2}} A_{\alpha,} \\
& g_{\alpha \beta}=h_{\omega \beta}-\frac{1}{\phi^{2}} A_{\alpha} A_{\beta},
\end{aligned}
$$

leading to the expression of the space-time interval in co-moving coordinates:

$$
\begin{aligned}
d s^{\prime \prime}= & -\frac{1}{\phi^{2}} d t^{2}+\frac{2}{\phi^{\prime 2}} A_{\alpha} d x^{\alpha} d t \\
& +\left(h_{\omega \beta}-\frac{1}{\phi^{2}} A_{\alpha} A_{\beta}\right) d x^{\alpha} d x^{\beta} .
\end{aligned}
$$

Co-moving coordinates have of course been discussed previously in nondyadic form by many authors (Ref. 11).

\section{Differentiation of Scalars, Vecfors, and Dyadics}

The various operations of differentiation in the 3-space orthogonal to a timelike congruence were introduced and fully discussed in Ref. 10. The purpose here is primarily to relate these operations to, and express them in terms of, partial derivatives with respect to a system of comoving coordinates.

Let $\psi\left(t, x^{\alpha}\right)$ be an arbitrary scalar function of such coordinates. For the proper time derivative, we have

$$
\dot{\psi} \equiv \frac{d \psi}{d \tau}=\frac{\partial \psi}{\partial x^{\alpha}} \dot{x}^{\alpha}+\frac{\partial \psi}{\partial t} \dot{t}=\phi_{\phi} \frac{\partial \psi}{\partial t},
$$

where the last equality results from $\dot{x}^{\alpha}=0$ and $\phi=\dot{t}$. For the gradient of $\psi$ orthogonal to the congruence, we have

$$
\begin{aligned}
\boldsymbol{\nabla} \psi & =\frac{\partial \psi}{\partial \boldsymbol{x}^{\alpha}} \boldsymbol{\nabla} x^{\alpha}+\frac{\partial \psi}{\partial t} \nabla t \\
& =\frac{\partial \psi}{\partial \boldsymbol{x}^{\alpha}} \mathbf{e}^{\alpha}+\frac{\dot{\psi}}{\phi} \mathbf{A}
\end{aligned}
$$

where we have used Eq. (25) and the relations $\mathbf{e}^{\alpha}=\nabla x^{\alpha}$ and $\mathbf{A}=\nabla t$. This may be rewritten as

$$
\boldsymbol{\nabla} \psi=\left(\frac{\partial \psi}{\partial x^{\alpha}}+\frac{\dot{\psi}}{\phi} A_{\alpha}\right) \mathbf{e}^{\alpha} \equiv \psi, \alpha \mathbf{e}^{\alpha},
$$

and the second equality serves to define the threedimensional comma derivative notation. (In general, it is not simply a partial derivative.) Dotting this equation with the dual vector $\mathbf{e}_{\beta}$ gives then

$$
\mathbf{e}_{\beta} \cdot \nabla \psi=\psi, \beta=\frac{\partial \psi}{\partial x^{\beta}}+\frac{\dot{\psi}}{\phi} A_{\beta},
$$

which clearly represents the change of $\psi$ orthogonal to the timelike congruence in the $\left(x^{\beta}, t\right) 2$-surface. $\psi_{, \alpha}$ is the intrinsic derivative of $\psi$ in the direction of $\mathbf{e}_{a}$. It is quickly verified that, for the special cases, $\psi=x^{\alpha}$ or $\psi=t$, Eq. (28) reproduces previous relations. The orthonormal components of the gradient, denoted by $\nabla_{a} \psi$, become

$$
\boldsymbol{\nabla}_{a} \psi=\mathbf{u}_{a} \cdot \boldsymbol{\nabla} \psi=\psi_{, a} \mathbf{e}^{\alpha} \cdot \mathbf{u}_{a}=\psi_{, \alpha a} \lambda^{\alpha},
$$

giving the change of $\psi$ along the spacelike congruence having $\mathbf{u}^{i}$ as unit tangent. Finally, for the total differential over a displacement $\mathbf{d x}$ in the orthogonal 3-space, we have

$$
d \psi=\nabla \psi \cdot \mathbf{d x}=\nabla \psi \cdot \mathbf{e}_{\kappa} \mathbf{e}^{\alpha} \cdot \mathbf{d} \mathbf{x}=\psi_{, \alpha} d x^{\alpha} .
$$


Time differentiation of vectors is defined in terms of the dot derivative such that for an arbitrary vector, $\mathrm{V}$,

$$
\dot{\mathbf{V}}=\left(\mathbf{V} \cdot \mathbf{u}_{a}\right)^{\bullet} \mathbf{u}^{a}=\dot{V}_{a} \mathbf{u}^{a}
$$

that is, it signifies the derivative with respect to proper time of the orthonormal components of $\mathrm{V}$ in any given triad system. It follows from this definition that $\dot{\mathbf{u}}_{a}=\mathbf{0}$ always. (In Ref. 10, this definition of dot differentiation occurred naturally by first differentiating intrinsically in the timelike $\mathbf{u}^{0}$ direction, and then "strangling" with the triad vectors.) The absolute derivative of the orthonormal triad vectors along the timelike congruence will be written

$$
\frac{\delta \mathbf{u}^{a}}{\delta \tau}=-\left(\mathbf{a} \cdot \mathbf{u}^{a}\right) \mathbf{u}^{0}+\boldsymbol{\omega} \times \mathbf{u}^{a},
$$

where $\mathbf{a}=\delta \mathbf{u}^{0} / \delta \tau$ is the acceleration of the timelike congruence, and $\omega$ is defined by this equation to agree with Ref. 10 and Eq. (B-6). The first term on the right generates Fermi-Walker transport, while $\omega$ describes any rotation of the triad relative to Fermi axes. As shown in Ref. 10, the triads may be introduced having any $\omega$ whatever. The absolute derivative of $\mathbf{V}$ can now be obtained, using its expansion, as

$$
\begin{aligned}
\frac{\delta \mathbf{V}}{\delta \tau} & =\frac{\delta}{\delta \tau}\left(V_{a} \mathbf{u}^{a}\right)-\frac{\delta V_{a}}{\delta \tau} \mathbf{u}^{n}+\mathbf{V}_{a} \frac{\delta \mathbf{u}_{a}}{\delta \tau} \\
& =-(\mathbf{a} \cdot \mathbf{V}) \mathbf{u}^{0}+\dot{\mathbf{V}}+\boldsymbol{\omega} \times \mathbf{V} .
\end{aligned}
$$

We note that the quantity $\dot{\mathbf{V}}+\boldsymbol{\omega} \times \mathbf{V}$ transforms vectorially under a change of basis vectors $u^{a}$ although its separate terms may not, and that its vanishing in any triad system is the necessary and sufficient condition for Fermi transport of $\mathbf{V}$.

It is verified in Appendix $B$ that the time derivatives of the natural basis vectors $\mathbf{e}^{\alpha}$ and $\mathbf{e}_{\alpha}$, associated with a co-moving coordinate system, are given by

$$
\begin{aligned}
& \dot{\mathbf{e}}^{\alpha}=-[\mathbf{S}-(\Omega-\boldsymbol{\omega}) \times \mathbf{I}] \cdot \mathbf{e}^{\alpha}, \\
& \dot{\mathbf{e}}_{\alpha}=\mathbf{e}_{a} \cdot[\mathbf{s}-(\Omega-\boldsymbol{\omega}) \times \mathrm{I}],
\end{aligned}
$$

where $S$ and $\Omega$ are the rate-of-strain dyadic and angular velocity vector of the timelike congruence. One result of these equations is

$$
\left(h_{\alpha \beta}\right)^{\bullet}=\left(\mathbf{e}_{a} \cdot \mathbf{e}_{\beta}\right)^{\bullet}=2 \mathbf{e}_{\alpha} \cdot s \cdot \mathbf{e}_{\beta},
$$

relating the time dependence of the orthogonal metric to S. Also, putting $\mathbf{V}=\mathbf{e}^{\kappa}$ in Eq. (33), we find for the absolute derivative

$$
\frac{\delta \mathbf{e}^{\alpha}}{\delta \tau}=-a^{\alpha} \mathbf{u}^{0}-(\mathbf{S}-\Omega \times \mathbf{I}) \cdot \mathbf{e}^{\alpha} .
$$

Dot differentiation of dyadics is similarly defined in terms of the variation of their orthonormal components. It follows that the absolute derivative of a dyadic $M$ is given by

$$
\frac{\delta M}{\delta \tau}=-a \cdot M u^{0}-M \cdot a u^{0}+\omega \times M-M \times \omega+\dot{M} .
$$

Turning to spatial differentiation of vectors we define, as in Ref. 10, the intrinsic vector differential operator D in terms of the gradients of the orthonormal components of a vector $\mathbf{V}$ in a given triad basis;

$$
\mathbf{D V}=\mathbf{D}\left(V_{a} \mathbf{u}^{a}\right)=\mathbf{D}\left(V_{a}\right) \mathbf{u}^{a}=\left(\nabla V_{a}\right) \mathbf{u}^{a} .
$$

The last equality follows because there is, of course, no distinction between $\mathbf{D}$ and $\boldsymbol{\nabla}$ when applied to scalars. By definition then, $\mathbf{D u}^{a} \equiv 0$ and $\mathbf{D}$ is a non-covariant operator involving essentially only comma derivatives, since we may write from Eq. (27)

$$
\mathbf{D} \bar{V}_{a}=\nabla \bar{V}_{a}=\left(\bar{V}_{a}\right)_{, \alpha} \mathbf{e}^{\tilde{a}}=\left(\bar{V}_{a}\right)_{, a} \dot{\lambda}^{\alpha} \mathbf{u}^{b},
$$

and so obtain for the orthonormal expansion of the quantity DV,

$$
\mathbf{D V}=\left(V_{a}\right)_{, \alpha} \lambda^{\alpha} \mathbf{u}^{b} \mathbf{u}^{a}
$$

We shall use the symbol $\nabla$ for the vector operator of 3 -space covariant differentiation so that

$$
\boldsymbol{\nabla} \mathbf{V}=\boldsymbol{\nabla}\left(V_{a} \mathbf{u}^{a}\right)=\left(\nabla V_{a}\right) \mathbf{u}^{a}+V_{a}\left(\nabla \mathbf{u}^{a}\right)=\mathbf{D V}+V_{a} \boldsymbol{\nabla} \mathbf{u}^{a}
$$

As brought out in Ref. 10, one of the advantages of orthonormal reference vectors is the fact that the anholonomic affinity

$$
\Gamma_{a}^{b} \cdot c=\mathbf{u}_{a} \cdot \nabla \mathbf{u}^{b} \cdot \mathbf{u}_{c},
$$

is antisymmetric on the last pair of indices rather than symmetric on first and last, as with the usual affinity. In three-dimensional space it has 9 components, instead of the 18 normally required, and so may be represented 
conveniently by a nonsymmetric dyadic. If we define the dyadic $\mathbf{N}$,

$$
\mathbf{N}=\frac{1}{2} \nabla \mathbf{u}^{a} \times \mathbf{u}^{a}
$$

to represent the affinity components, we find by crossing $\mathbf{u}^{b}$ from the right, using the orthonormality relations, Eq. (1), and the identity, (A-8), that

$$
\boldsymbol{\nabla} \mathbf{u}^{b}=-\mathbf{N} \times \mathbf{u}^{b},
$$

so that the gradient of each triad vector is expressed by $\mathbf{N}$. The affinity components are related to those of $\mathbf{N}$ by

$$
\Gamma_{a}^{\cdot b}=\mathbf{u}_{a} \cdot \nabla \mathbf{u}^{b} \cdot \mathbf{u}_{c}=-\mathbf{u}_{a} \cdot \mathbf{N} \times \mathbf{u}^{b} \cdot \mathbf{u}_{c}=-\boldsymbol{\varepsilon}^{d b c} N_{a d},
$$

although we shall consistently prefer the dyadic representation. [The use of a dyadic to represent the anholonomic affinity of orthonormal triads is expedient for its simplicity and economy of notation. It must be observed however that, since it is an affinity, $\mathbf{N}$ will not transform under an arbitrary change of basis vectors according to the usual linear, homogeneous law for dyadics. The general transformation law for $\mathbf{N}$ is derived in Ref. 10 and has the familiar inhomogeneous term depending on derivatives of the transformation matrix, as expected for an affinity. These same remarks apply with equal force to the use of a vector, $\omega$, to represent the angular velocity of the triads. Its transformation properties are also obtained in Ref. 10, and it is shown that any values for the $\omega$ field, including zero, may be obtained simply by an appropriate choice of triads. In practice, we almost always adopt a particular intrinsically defined set of basis triads and stick then to sets related to these by a carefully delimited process of "3-space rotation" under which $\mathbf{N}$ and $\boldsymbol{\omega}$ transform linearly and homogeneously. The situation is perfectly analogous to the use of tensorlike notation for the usual affinity $\Gamma_{v \sigma}^{\mu}$, whose transformation law is nontensorial and seldom directly used.]

We also point out a notational change from Ref. 10 where the dyadic affinity was denoted by $\mathbf{N}^{\star}$, and the bare symbol $\mathbf{N}$ represented only its symmetric part (up to a trace). This notation has proved slightly clumsy, since we deal almost exclusively with the unsymmetrized object. Accordingly, in this report, $\mathbf{N}$ replaces the $\mathbf{N}^{\star}$ of Ref. 10. If and when a symbol is required for its symmetric part, we shall use $\mathbf{N}^{s}=1 / 2\left(\mathbf{N}+\mathbf{N}^{T}\right)$ as in Appendix A.
The explanation of the smaller number of independent components of the affinity is now seen from the fact that only the 9 components in the curls of the three orthonormal basis vectors are actually involved. From the identity

$$
\nabla \times \mathbf{I}=\nabla \times\left(\mathbf{u}^{a} \mathbf{u}^{a}\right)=0,
$$

and using Eq. (A-19), we can obtain the relation

$$
\mathbf{N}=-\mathbf{u}^{a}\left(\nabla \times \mathbf{u}^{a}\right)+\frac{1}{2}\left[\mathbf{u}^{a} \cdot\left(\nabla \times \mathbf{u}^{a}\right)\right] \mathbf{l},
$$

expressing $\mathbf{N}$ in terms of the curls alone. Writing the antisymmetric part of $\mathbf{N}$ as a vector $\mathbf{n}$ (Appendix $\mathrm{A}$ ),

$$
\frac{1}{2}\left(\mathbf{N}-\mathbf{N}^{T}\right)=-\mathbf{n} \times \mathbf{l},
$$

we find several equivalent expressions for $\mathbf{n}$,

$$
\mathbf{n}=-\frac{1}{2} \mathbf{u}^{a} \times\left(\nabla \times \mathbf{u}^{a}\right)=\frac{1}{2} \mathbf{u}^{a} \cdot \nabla \mathbf{u}^{a}=-\frac{1}{2}\left(\nabla \cdot \mathbf{u}^{a}\right) \mathbf{u}^{a} .
$$

According to the last equality, each triad component of $\mathbf{n}$ thus represents the covariant divergence of that same triad vector. In Ref. 10, $n$ was denoted $L$.

Returning to Eq. (41), we may now write the covariant derivative of $\mathbf{V}$ in terms of $\mathbf{N}$ as

$$
\nabla \mathbf{V}=\mathbf{D V}-\mathbf{V}_{a} \mathbf{N} \times \mathbf{u}^{a}=\mathbf{D V}-\mathbf{N} \times \mathbf{V}
$$

The generalization to covariant differentiation of dyadics is immediate. Using the identity (A-19), we have for an arbitrary dyadic $\boldsymbol{M}$

$$
\boldsymbol{\nabla} \times \mathbf{M}=\mathbf{D} \times \mathbf{M}+(\operatorname{Tr} \mathbf{N}) \mathbf{M}-\mathbf{N}^{T} \cdot \mathbf{M}-\underset{\times}{\mathbf{N}^{T \times} \mathbf{M}},
$$

where the double cross notation is defined in Appendix A.

\section{The Dyadic Equations for Rigid Reference Frames in Einstein Space}

A general timelike congruence may be described at each point by its absolute acceleration vector $\mathbf{a}$, angular velocity vector $\Omega$, and symmetric rate-of-strain dyadic 5. The precise definitions of these quantities and the general dyadic equations they satisfy are given in Appendix B. Here, we write the equations specialized to rigid congruences in Einstein spaces. The notation is defined in Appendix A. 
Following Born, the rigid congruence is defined by $S=0$, so that all rates of shear and expansion vanish. In spacetime, this is equivalent to constraining the world lines of the timelike congruence to have constant orthogonal separations. The geometrical consequence of this constraint is revealed by Eq. (36); the orthogonal 3-space metric on a rigid body in co-moving coordinates is constant throughout time, $\left(h_{\alpha \beta}\right)^{\circ}=0$. By adopting orthonormal basis triads which co-rotate with the rigid body $(\omega=\Omega)$ and are thus fixed in it, we have in Appendix B the dyadic $S^{\star}=0$ and, from Eq. (B-16), we see that the anholonomic affinity is time independent: $\dot{\mathbf{N}}=0$. In this situation it is permissible to replace the many local orthogonal 3-spaces with the concept of a single metric quotient space: the three-dimensional manifold of comoving coordinates $x^{\alpha}$. Geometrically, we may picture the quotient space as a reduction of space-time obtained when all events lying on each world line of the timelike congruence are identified. The orthogonal metric $h_{\alpha \beta}$ and the affinity $\mathbf{N}$ are then applied to this quotient space.

Eq. (B-17) becomes the curvature equation for a threedimensional metric space:

$$
\nabla \times \mathbf{N}=-\frac{1}{2} \mathbf{N}_{\times}^{T} \mathbf{N}+\mathbf{E} .
$$

The symmetric dyadic $E$ is the conservative dyadic of the quotient space, satisfying the Bianchi identity

$$
\boldsymbol{\nabla} \cdot \mathbf{E}=\mathbf{0} .
$$

Its diagonal elements are the Riemannian curvatures of the quotient space, based on the orthonormal triad vectors, while all six components are sufficient to express the complete 3 space curvature tensor, the exact relation being exhibited in Eq. (B-19). Since $\dot{\mathbf{N}}=0$, we can apply the commutation relation equation (54) to (51) to show that

$$
\dot{\mathbf{E}}=0,
$$

independently of the coordinate system employed. In co-moving coordinates this is, of course, seen as a trivial consequence of $\left(h_{\alpha \beta}\right)^{\bullet}=0$.

The commutation rules for covariant and time differentiation in the quotient space are somewhat unfamiliar, in general, as a result of the use of anholonomic reference systems. And although the geometry of the quotient space is constant in time, we may have time-dependent objects in the space; for these the order of space and time differentiation is of consequence. Letting $\psi, \mathbf{V}$, and $\mathbf{M}$ symbolize an arbitrary scalar, vector, and dyadic, we have

$$
\left.\begin{array}{rl}
(\nabla \psi)^{\bullet}-\nabla(\dot{\psi}) & =\mathbf{a} \dot{\psi}, \\
(\nabla \mathbf{V})^{\bullet}-\nabla(\dot{\mathbf{V}}) & =\mathbf{a} \dot{\mathbf{V}}, \\
(\nabla \times \mathbf{M})^{\bullet}-\nabla \times(\dot{\mathbf{M}}) & =\mathbf{a} \times \dot{\mathbf{M}} .
\end{array}\right\}
$$

Note that for any time-independent quantity, these operations do commute; this is a special property of rigid congruences.

Commutation of the covariant operator $\boldsymbol{\nabla}$ with itself is also somewhat unusual, except when the angular velocity $\Omega$ of the rigid body vanishes. (In this latter case the 4-velocity of the body is derivable from a scalar potential and its world lines are the orthogonal trajectories of a family of hypersurfaces in space-time. Consequently, the quotient space becomes isomorphic to all the members of a family of immersed Riemannian 3spaces, which could be parameterized by $t$.) Samples of the space-space commutation relations are

$$
\begin{aligned}
\nabla \times \nabla \psi & =2 \dot{\psi} \boldsymbol{\Omega}, \\
\nabla \cdot(\nabla \times \mathbf{V}) & =2 \boldsymbol{\Omega} \cdot \dot{\mathbf{V}}, \\
\nabla \times(\nabla \mathbf{V}) & =-\mathbf{E} \times \mathbf{V}+2 \boldsymbol{\Omega} \dot{\mathbf{V}}
\end{aligned}
$$

Next we impose the condition for Einstein space in the form

$$
G_{\mu \nu}=R_{\mu \nu}-\frac{1}{2} R g_{\mu \nu}=\Lambda g_{\mu \nu}
$$

where $\Lambda=-1 / 4 R$ is the cosmological constant. In orthonormal components this equation leads to the following expressions for the curvature quantities of Eq. (B-12):

$$
\begin{aligned}
& \mathbf{T}=\frac{1}{2} \Lambda \mathbf{I}, \\
& \mathbf{t}=0, \\
& \rho=\frac{1}{2} \Lambda .
\end{aligned}
$$

There are 10 of the 20 curvature components specified by these equations. The remaining 10 are represented in the two symmetric, traceless dyadics $\mathbf{A}$ and $\mathbf{B}$, which are 
arbitrary. We note that since $\mathbf{t}=0$, we have $\mathbf{B}^{\star}=\mathbf{B}^{\star T}$ $=\mathbf{B}$ and, using the relations in Eq. (B-13), we find

$$
\mathbf{P}=\mathbf{Q}=\mathbf{A}-\frac{1}{3} \Lambda \mathbf{}, \quad \operatorname{Tr} \mathbf{P}=\operatorname{Tr} \mathbf{Q}=-\Lambda .
$$

The curvature of the quotient space is related to the space-time curvature by a generalized Gauss equation, which from Eq. (B-18) becomes

$$
\mathbf{E}=-\mathbf{A}+\frac{1}{3} \Lambda \mathbf{I}-3 \Omega \Omega .
$$

This equation, together with Eq. (53), has important consequences for allowed rigid motions, which will be discussed fully in later sections.

As a vector field in the quotient space, the acceleration of the rigid body satisfies [from Eq. (B-14)] the dyadic equation

$$
\nabla \mathbf{a}=-\mathbf{a} \mathbf{a}+\Omega \Omega-\dot{\Omega} \times \mathbf{I}+\mathbf{A}-\left(\Omega^{2}+\frac{1}{3} \Lambda\right) \mathbf{I},
$$

The trace of this equation reads

$$
\boldsymbol{\nabla} \cdot \mathbf{a}=-a^{2}-2 \Omega^{2}-\Lambda,
$$

and its antisymmetric part is

$$
\nabla \times \mathbf{a}=2 \dot{\mathbf{\Omega}} .
$$

This last equation is, in effect, the integrability condition for the potential equation for a [Eq. (17)], which here simplifies to

$$
\phi \mathbf{a}=-\nabla_{\phi}+\dot{\mathbf{A}} .
$$

The corresponding set of equations (B-15) for the angular velocity or vorticity field becomes

$$
\begin{aligned}
\nabla \boldsymbol{\Omega} & =-2 \mathbf{a} \boldsymbol{\Omega}+(\mathbf{a} \cdot \boldsymbol{\Omega}) \mathbf{I}+\mathbf{B}, \\
\boldsymbol{\nabla} \cdot \boldsymbol{\Omega} & =\mathbf{a} \cdot \boldsymbol{\Omega}, \\
\boldsymbol{\nabla} \times \boldsymbol{\Omega} & =2 \boldsymbol{\Omega} \times \mathbf{a},
\end{aligned}
$$

with the potential equation

$$
2 \phi \Omega=\nabla \times A .
$$

The gravitational field equations, or Bianchi identities [Eqs. (B-26) to (B-28)] have Maxwellian-like forms, but are, of course, dyadic equations:

$$
\begin{aligned}
& \nabla \times \mathbf{A}-\dot{\mathbf{B}}=-\mathbf{a} \times \mathbf{A}+\mathbf{A} \times \mathbf{a}-\Omega \times \mathbf{B}-2 \mathbf{B} \times \Omega, \\
& \nabla \times \mathbf{B}+\dot{\mathbf{A}}=-\mathbf{a} \times \mathbf{B}+\mathbf{B} \times \mathbf{a}+\Omega \times \mathbf{A}+2 \mathbf{A} \times \Omega .
\end{aligned}
$$

The antisymmetric parts of these two equations are the vector equations:

$$
\begin{aligned}
& \boldsymbol{\nabla} \cdot \mathbf{A}=-3 \boldsymbol{\Omega} \cdot \mathbf{B}, \\
& \boldsymbol{\nabla} \cdot \mathbf{B}=3 \boldsymbol{\Omega} \cdot \mathbf{A} .
\end{aligned}
$$

Finally, since we have specialized to Einstein space, the contracted Bianchi identities are trivial. They merely insist on the constancy of the cosmological constant;

$$
\dot{\Lambda}=\nabla \Lambda=0 \text {. }
$$




\section{RIGID MOTION IN SPACE-TIMES OF CONSTANT CURVATURE}

The problem of rigid motions in flat space-time was thoroughly treated by G. Herglotz (Ref. 2) and F. Noether (Ref. 3), and the generalization to constant curvature manifolds introduces virtually nothing new. For completeness, we simply summarize the results of these and a few subsequent authors on this problem, expressing them in the dyadic notation. The only original contribution here is a derivation of the quotient space metrics intrinsic to a rotating and accelerating rigid body in the space-time of constant curvature.

Setting the conformal curvature dyadics $\mathbf{A}$ and $\mathbf{B}$ to zero, we obtain the equations appropriate to a space-time of constant curvature, $K=1 / 3 \Lambda$. Combining Eqs. (53) and (61), we see that

$$
\Omega \dot{\Omega}+\dot{\Omega} \Omega=0,
$$

and since the trace of this equation gives $2 \Omega \cdot \dot{\Omega}=\left(\Omega^{2}\right)^{\circ}$ $=0$, we find by dotting $\Omega$ through that

$$
\dot{\mathbf{\Omega}}=\mathbf{0} \text {. }
$$

Since the equations are written in body-fixed axes, this result shows immediately that the angular velocity is a fixed vector in the body; however, since $\dot{\boldsymbol{\Omega}}+\boldsymbol{\omega} \times \boldsymbol{\Omega}$ also vanishes $(\omega=\Omega)$, we find that the angular velocity vector is fixed in Fermi propagated axes as well [Eq. (33)]. We note also for later use that Eq. (64) now reads simply, $\boldsymbol{\nabla} \times \mathbf{a}=\mathbf{0}$.

Using the fact that space and time derivatives of $\Omega$ will commute, it follows from time differentiating Eq. (67) that $(a \cdot \Omega)^{\bullet}=0$. Then treating Eq. (66) similarly, one gets the dyadic equation

$$
\dot{\mathrm{a}} \Omega=0 .
$$

Thus, if the angular velocity does not vanish, we must have $\dot{\mathbf{a}}=0$ so that the acceleration vector is body-fixed also. The two equations now satisfied by a when $\Omega \neq 0$, (i.e., $\nabla \times \mathbf{a}=\dot{\mathbf{a}}=0$ ), together with the constraint of rigidity $\mathbf{S}=0$, are the necessary and sufficient conditions for an isometric or Killing vector congruence. Thus, the Herglotz-Noether theorem applies to all space-times of constant curvature. The full 10-parameter set of isometries exists for these spaces and is well known; Herglotz (Ref. 2), in particular, has given a detailed treatment of the timelike isometries in flat space-time.
The curvature of the spatial geometry in a rotating rigid body depends on the angular velocity, as shown by the Gauss equation (61) for the 3-space curvature dyadic E,

$$
\mathbf{E}=-3 \Omega \Omega+\frac{1}{3} \Lambda \mathbf{l}
$$

The scalar curvature of the space is given by $-2 \operatorname{Tr} E$ and so has the value $-2\left(\Lambda-3 \Omega^{2}\right)$. The metric of the quotient 3 -space on a rotating rigid disk has been a subject of occasional interest in the literature of relativity; several references to early literature are given in the discussion by Berenda (Ref. 12). In constant curvature space-time, the most general rotating and accelerating rigid body is a typical example of a situation providing its own intrinsic co-moving coordinates, and the quotient metrics are easily obtained with this approach.

For the rotating body, three intrinsic scalars may exist: $a^{2}, \Omega^{2}$, and $a \cdot \Omega$, all of which must be time independent as we have shown, so that co-moving coordinates may be constructed from them. The gradients of these scalars are obtained directly from Eqs. (62) and (66) as

$$
\begin{aligned}
\nabla a^{2}= & 2(\nabla \mathbf{a}) \cdot \mathbf{a}=-2\left(a^{2}+\Omega^{2}+\frac{1}{3} \Lambda\right) \mathbf{a} \\
& +2(\mathbf{a} \cdot \Omega) \Omega, \\
\nabla \Omega^{2}= & -4\left(\Omega^{2}\right) \mathbf{a}+2(\mathbf{a} \cdot \Omega) \Omega, \\
\nabla(\mathbf{a} \cdot \Omega)= & (\nabla \mathbf{a}) \cdot \Omega+(\nabla \Omega) \cdot \mathbf{a} \\
= & -2(\mathbf{a} \cdot \Omega) \mathbf{a}-\frac{1}{3} \Lambda \Omega,
\end{aligned}
$$

and these equations can be combined to express the acceleration vector as a gradient in two ways;

$$
\begin{aligned}
\mathbf{a} & =-\frac{1}{4} \nabla \ln \left[a^{2}-\Omega^{2}+\frac{1}{3} \Lambda\right]^{2} \\
& =-\frac{1}{4} \nabla \ln \left[(a \cdot \Omega)^{2}+\frac{1}{3} \Lambda \Omega^{2}\right] .
\end{aligned}
$$

The last equality results in the integral

$$
(a \cdot \Omega)^{2}+\frac{1}{3} \Lambda \Omega^{2}=k^{2}\left(a^{2}-\Omega^{2}+\frac{1}{3} \Lambda\right)^{2},
$$


revealing that the three scalars are not independent, so that only two intrinsic coordinates can actually be constructed. Since space-times of negative constant curvature have very peculiar physical properties, we shall here consider $\Lambda \supseteq 0$ only. By the form of Eq. (78), the integration constant is then required to be positive, so is written $k^{2}$, and we take $k \geq 0$.

From Eq. (68), we note that $\Omega \cdot(\nabla \times \Omega)=0$. This is the integrability condition allowing $\Omega$ to be derived from a scalar potential. Reworking the previous gradient equations to solve for $\Omega$, we can find the explicit expression

$$
\Omega=\frac{\Omega^{2}}{2(\mathfrak{a} \cdot \Omega)} \nabla \ln \left[\frac{\Omega^{2}}{\left(a^{2}-\Omega^{2}+1 / 3 \Lambda\right)^{2}}\right] .
$$

If we define the vector $a_{\perp}$ as the component of the acceleration perpendicular to the angular velocity

$$
\mathbf{a}_{\perp} \equiv \mathbf{a}-\frac{(\mathbf{a} \cdot \boldsymbol{\Omega})}{\mathbf{\Omega}^{2}} \boldsymbol{\Omega},
$$

we find from Eqs. (77) and (79) that

$$
\mathbf{a}_{\perp}=\frac{1}{4} \nabla \ln \left[\frac{\left(a^{2}-\Omega^{2}+1 / 3 \Lambda\right)^{2}}{\left(\Omega^{2}\right)^{2}}\right] .
$$

It is now clear that two especially convenient intrinsic coordinates are obtained by taking one of them to be some function of the argument of the logarithm in Eq. (79), and the other a function of the argument in Eq. (81). For, as shown by Eqs. (79) and (81), the gradient vectors of such coordinates will be orthogonal, leading to an orthogonal metric. We select the functions by keeping in mind the flat space-time example of the rigid disk rotating about a fixed axis, and requiring the general metric to reduce in that limit to a familiar form in cylindrical co-moving coordinates.

This is accomplished for the argument in Eq. (79) by setting

$$
\frac{\Omega^{2}}{\left(a^{2}-\Omega^{2}+1 / 3 \Lambda\right)^{2}}=\frac{k^{2}}{\alpha^{2}} \sin ^{2}\left[\alpha\left(\frac{1}{\omega_{11} k}-z\right)\right],
$$

where $\alpha \equiv(1 / 3 \Lambda)^{1 / 2}, \omega_{01}$ is a new constant, and $z$ is introduced as the coordinate whose surfaces have $\Omega$ as normal vector. In flat space-time $(\Lambda=\alpha=0)$, this reduces to

$$
\frac{\left(a^{2}-\Omega^{2}\right)^{2}}{\Omega^{2}}=\frac{\omega_{0}^{2}}{\left(1-\omega_{0} k z\right)^{2}}, \quad \Lambda=0 .
$$

But in addition for the fixed axis disk a $\Omega=0$ everywhere, which from Eq. (78) requires $k=0$, so that

$$
\frac{\left(a^{2}-\Omega^{2}\right)^{2}}{\Omega^{2}}=\omega_{0}^{2}, \quad \Lambda=k=0 .
$$

On the axis of the disk $a^{2}=0$, and so for this special case $(1)_{0}$ is identified as the angular velocity on the axis.

The coordinate $r$ is introduced by putting

$$
\frac{a^{2}-\Omega^{2}+1 / 3 \Lambda}{\Omega^{2}}=-\frac{1}{2 k^{2}}\left[-1+\beta \cos \left(2 k \omega_{0} r\right)\right],
$$

where $\beta \equiv\left(1+4 k^{2}\right)^{1 / 2}$, and $\mathbf{a}_{\perp}$ is thus orthogonal to the coordinate surfaces $r=$ constant. In the rigid disk limit, this becomes

$$
\frac{a^{2}-\Omega^{2}}{\Omega^{2}}=-1+\omega_{0}^{2} r^{2}, \quad \Lambda=k=0,
$$

and the axis, $a^{\prime \prime}=0$, is properly located at $r=0$. Now solving simultaneously Eqs. (78), (82), and (83), we express the intrinsic scalars for the general case in terms of these intrinsic coordinates;

$$
\begin{aligned}
a^{2} & =a_{\perp}^{2}+\frac{(\mathrm{a} \cdot \Omega)^{2}}{\Omega^{2}} \\
a_{\perp}^{2} & =\alpha^{2} \beta^{2} R^{-2} \sin ^{2}\left(2 k_{\omega_{0}} r\right) \csc ^{2} Z, \\
\Omega^{2} & =4 \alpha^{2} k^{2} R^{-2} \csc ^{2} Z \\
(\mathrm{a} \cdot \Omega)^{2} & =4 \alpha^{4} k^{2} R^{-2} \csc ^{2} Z \cot ^{2} Z
\end{aligned}
$$

where

$$
\begin{aligned}
& R \equiv-1+\beta \cos \left(2 k_{\omega_{0}} r\right), \\
& Z \equiv \alpha\left(\frac{1}{\omega_{0} k}-z\right) .
\end{aligned}
$$

By differentiating Eqs. (82) and (83), substituting in Eqs. (79) and (81), and using the expressions in Eq. (84), we find

$$
\Omega=|\Omega| \nabla z
$$

showing $\nabla z$ as a unit vector, and

$$
\mathbf{a}_{\perp}=-\left|\mathbf{a}_{\perp}\right|\left(\frac{k_{\omega_{11}}}{\alpha}\right) \sin Z \nabla r .
$$


A third intrinsic coordinate does not exist; however, applying the dyadic equations [(62) and (66)] for $\nabla \mathbf{a}$ and $\nabla \Omega$, we easily prove that

$$
(\mathbf{a} \times \Omega) \cdot[\nabla \times(a \times \Omega)]=0,
$$

so that a scalar potential also exists for this vector which is orthogonal to both $\Omega$ and $\mathbf{a}_{\perp}$. Let the potential be denoted by $\bar{\theta}$ and write

$$
\begin{aligned}
\mathbf{a} \times \Omega= & \left|\mathbf{a}_{\perp}\right||\Omega|\left(\frac{\beta}{\omega_{0}}\right) \frac{\sin \left(2 k \omega_{\omega_{0}} r\right)}{(2|\boldsymbol{R}|)^{1 / 2}}\left(\frac{k \omega_{0}}{\alpha}\right) \\
& \times \sin Z \xi(r, \bar{\theta}, z) \nabla \bar{\theta} .
\end{aligned}
$$

Here $\xi(r, \bar{\theta}, z)$ is an arbitrary scale factor. The rest of the coefficient of $\nabla \bar{\theta}$ is written separately for the following reason: if we calculate the curl of both sides of this equation, we find that

$$
\nabla \xi \times \nabla \bar{\theta}=0
$$

implying that $\nabla \xi$ is collinear with $\nabla \bar{\theta}$ so that $\xi$ must be a function of $\bar{\theta}$ only. Consequently, we may introduce a new potential $\theta$ defined by

$$
\xi(\bar{\theta}) \nabla \bar{\theta}=\nabla \theta,
$$

and have

$$
\begin{aligned}
\mathbf{a} \times \Omega= & \left|\mathbf{a}_{1}\right||\Omega|\left(\frac{\beta}{\omega_{0}}\right) \frac{\sin \left(2 k_{\omega_{0}} r\right)}{(2|R|)^{1 / 2}}\left(\frac{k_{\omega_{0}}}{\alpha}\right) \\
& \times \sin Z \nabla \theta,
\end{aligned}
$$

where $\theta$ is now adopted for the third orthogonal coordinate.

The gradients of the coordinates constitute the natural basis vector triad $\mathbf{e}^{\alpha}$, as defined in Section II-B, and putting

$$
\mathbf{e}^{1}=\boldsymbol{\nabla r}, \quad \mathbf{e}^{2}=\boldsymbol{\nabla} \theta, \quad \mathbf{e}^{3}=\boldsymbol{\nabla} z,
$$

we use Eqs. (85), (86), and (87) to determine the dual set as

$$
\begin{aligned}
& \mathbf{e}_{\mathbf{1}}=-\left(\frac{k_{\omega_{0}}}{\alpha}\right) \sin Z \frac{\mathbf{a}_{\perp}}{\left|\mathbf{a}_{\perp}\right|}, \\
& \mathbf{e}_{2}=\left(\frac{k_{\omega_{0}}}{\alpha}\right) \sin Z\left(\frac{\beta}{\omega_{0}}\right) \frac{\sin \left(2 k \omega_{0} r\right)}{(2|R|)^{1 / 2}} \frac{\mathbf{a} \times \Omega}{|\mathbf{a} \times \Omega|}, \\
& \mathbf{e}_{3}=\frac{\Omega}{|\Omega|},
\end{aligned}
$$

satisfying the inversion $\mathbf{e}^{\alpha} \cdot \mathbf{e}_{\beta}=\delta_{\beta}^{\alpha}$. Using $h_{\alpha \beta}=\mathbf{e}_{\alpha} \cdot \mathbf{e}_{\beta}$ the general quotient space metric for a rotating, accelerating, rigid body in constant curvature space-time becomes

$d l^{2}=\left(\frac{k_{\omega_{0}}}{\alpha}\right)^{2} \sin ^{2} Z\left\{d r^{2}+\left(\frac{\beta}{\omega_{0}}\right)^{2} \frac{\sin ^{2}\left(2 k \omega_{0} r\right)}{2|\bar{R}|} d \theta^{2}\right\}+d z^{2}$.

Limits are required on the ranges of both $z$ and $r$ coordinates; first for $z$

$$
0<Z=\alpha\left(\frac{1}{\omega_{0} k}-z\right)<\pi
$$

since not only is the metric singular at these limits, but from Eq. (84) all the intrinsic scalars approach infinity here. The limits on the $r$ coordinate result from two considerations: the sine function in the coefficient of $d \theta^{2}$ requires

$$
0 \leq 2 k_{\omega_{0}} r \leq \pi,
$$

but this range is subdivided by the vanishing of the quantity $R$ in the denominator at $\cos \left(2 k_{\omega_{0}} r\right)=\beta^{-1}$. Since the intrinsic scalars become infinite at this point, it is best to give two different metrics for each range.

The first becomes

I. $\quad 0 \leq r \leq \frac{1}{2 k_{\omega_{0}}} \cos ^{-1}\left(\frac{1}{\beta}\right)$

$$
\begin{aligned}
d l^{2}= & \left(\frac{k_{\omega_{0}}}{\alpha}\right)^{2} \sin ^{2} Z\left\{d r^{2}+\left(\frac{\beta}{\omega_{0}}\right)^{2}\right. \\
& \left.\times \frac{\sin ^{2}\left(2 k_{\omega_{0}} r\right)}{2\left[\beta \cos \left(2 k_{\omega_{0}} r\right)-1\right]} d \theta^{2}\right\}+d z^{2} .
\end{aligned}
$$

By letting $\alpha \rightarrow 0$, we find for flat space-time

$$
\begin{aligned}
d l^{2}= & \left(1-\omega_{0} k z\right)^{2}\left\{d r^{2}+\left(\frac{\beta}{\omega_{0}}\right)^{2}\right. \\
& \left.\times \frac{\sin ^{2}\left(2 k_{\omega_{0}} r\right)}{2\left[\beta \cos \left(2 k_{\omega_{0}} r\right)-1\right]} d \theta^{2}\right\}+d z^{2}, \quad \Lambda=0 .
\end{aligned}
$$

And if we further let $k$ vanish so that $\mathbf{a} \cdot \Omega=0$, we get

$$
d l^{2}=d r^{2}+\frac{r^{2}}{1-\omega_{0}^{2} r^{2}} d \theta^{2}+d z^{2}, \quad \Lambda=k=0,
$$


which is the quotient metric on a fixed-axis rotating rigid disk in the form obtained by Berenda (Ref. 12). Compare also the discussion by M $\phi$ ller (Ref. 13). In Eq. (92), the $r$ coordinate ranges over the finite interval $0 \leq r \leq\left(\omega_{0}\right)^{-1}$; it is easily seen from Eq. (84) specialized to this case that both $a_{\perp}^{2}$ and $\Omega^{2}$ increase without limit as $r \rightarrow\left(\omega_{0}\right)^{-1}$, which thus corresponds to the greatest possible extent of the disk, the boundary moving at light velocity.

In order to write the metric for the second allowed range, it is desirable to introduce a new coordinate $\bar{r}$ according to

$$
2 k_{\omega, 1} \bar{r}=\pi-2 k_{\omega_{0},} r
$$

so that

$$
\text { II. } \begin{aligned}
0 \leq \bar{r} \leq & \frac{1}{2 k_{\left(\omega_{10}\right.}} \cos ^{-1}\left(-\frac{1}{\beta}\right) \\
d l^{2}= & \left(\frac{k_{\omega_{11}}}{\alpha}\right)^{2} \sin ^{2} Z\left\{d \bar{r}^{2}+\left(\frac{\beta}{\omega_{10}}\right)^{2}\right. \\
& \left.\times \frac{\sin ^{2}\left(2 k_{\omega_{0}} \bar{r}\right)}{2\left[\beta \cos \left(2 k_{\omega_{0}} \bar{r}\right)+1\right]} d \theta^{2}\right\}+d z^{2} .
\end{aligned}
$$

The limiting case $\Lambda=k=0$ is of little interest here, since it can be shown from Eq. (84) that it corresponds to vanishing angular velocity in flat space-time, and consequently a flat quotient space results. This completes our discussion of rotating and accelerating rigid frames in space-times of constant curvature, that is to say, of the isometries of these spaces and the geometry of their quotient spaces.

The irrotational rigid motions are physically more interesting in these spaces, as at least some degrees of freedom are present. Actually, however, there is not much need for analytical investigation of these motions, since a simple geometrical construction for them exists and elucidates their features better than a set of equations could. For flat space-time, this construction was mentioned by Herglotz (Ref. 2) and analyzed in detail by Fokker (Ref. 14); a good discussion of the general case is found in Ref. 9.

When $\Omega=0$, the unit tangent vector to any timelike congruence is derivable from a scalar potential. The world lines of the irrotational rigid congruence are consequently the orthogonal trajectories of a family of threedimensional hypersurfaces in space-time. The rigidity of the congruence ensures that all these hypersurfaces, any of which may be identified with the quotient space, will have identical metric properties; the congruence can indeed be viewed simply as an isometric mapping of each hypersurface on all the others. It is further easily shown that the hypersurfaces must be totally geodesic. A simple example of this last property is found in flat space-time, where the Gauss equation (61) shows that $\mathbf{E}=0$, so that the quotient space and all the hypersurfaces must also be flat.

In flat space-time, two closely successive spacelike hypersurfaces $\mathcal{O}_{3}$ clearly intersect in a spacelike 2 -flat $\mathcal{O}_{2}$; a third successive hypersurface cuts this in a spacelike straight line $\varrho_{1} ;$ a fourth determines a point $\Theta_{0}$. The locus (spacelike) of all such points $\mathscr{O}_{0}$ in the space-time is the so-called edge-of-regression $\mathcal{C}$ of the congruence. The tangents, osculating planes and osculating 3-flats to $\Theta$ are the $\mathcal{O}_{1}^{\prime}$ 's, $\mathcal{C}_{2}$ 's, and $\mathcal{C}_{3}$ 's, respectively. Each rigid normal congruence determines a $\mathcal{O}$, and conversely. The three degrees of freedom of nonrotating rigid frames in flat space-time could explicitly be displayed in a parametric set of equations for 0 .

Since each point $\omega_{0}$ may be mapped into one given hypersurface, the line $\circlearrowright$ also exists as a locus in the quotient space. The $O_{1}$ 's and $C_{2}$ 's also may be mapped there, their envelope being the boundary of the body, in the sense that no point of the body inside it ever has infinite acceleration. The edge-of-regression $O$ lies in the boundary.

As mentioned by Boyer, this same construction goes through when the space-time is of constant curvature, as the subspaces $O_{3}, O_{2}, O_{1}$ are again each in turn totally geodesic. In Section V, when general Einstein space is considered, we will see that the present three degrees of freedom for irrotational motion are reduced to one, and that even this freedom only occurs in metrics which allow the rigid congruence lines to lie in uniformly curved timelike 2-spaces. The edge of regression $\mathcal{O}$ becomes then rather a 3-surface cutting across all these 2-spaces, and every congruence line has infinite acceleration at some point. 


\section{GEODESIC RIGID FRAMES}

Since both $\dot{\mathbf{a}}=0$ and $\nabla \times \mathbf{a}=0$ are trivially true when $a=0$, it is clear that geodesic rigid congruences are a subclass of isometric motions, so that inclusion of this section violates our intention not to discuss isometries in detail. Actually, the only reason for considering these motions is to dispose of them, for as mentioned by Boyer (Ref. 9) and as we shall show, the geodesic rigid reference frame is impossible in any gravitational field outside matter. This result is perhaps not too surprising; in fact, when the cosmological constant is not present, the proof is very quick. It is surprising how relatively complicated the proof becomes with inclusion of $\Lambda$. In the presence of real matter, of course, geodesic rigid motion is possible. The static Einstein universe is the simplest example having no angular velocity, while Gödel's universe is the simplest with rotation; both of these models have a constant matter density and require $\Lambda \neq 0$, in addition.

For the geodesic rigid frame, we put $a=0$ throughout the equations of Section II-D. From the time-space commutation relations [Eq. (54)], we see that these operations now always commute. The set of acceleration equations [Eqs. (62) to (64)] gives

$$
\begin{aligned}
& \Omega^{2}=-\frac{1}{2} \Lambda \\
& \dot{\Omega}=0 \\
& \mathbf{A}=-\Omega \Omega+\frac{1}{3}\left(\Omega^{2}\right) \mathbf{l}
\end{aligned}
$$

and from Eq. (66)

$$
\nabla \Omega=\mathbf{B} .
$$

Time differentiating Eqs. (96) and (97), we find $\dot{\mathbf{A}}=\dot{\mathbf{B}}=0$, and all quantities are time independent as expected for an isometry. Clearly if $\Lambda$ vanishes, $\Omega=0$, and the vanishing of the gravitational field dyadics $\mathbf{A}$ and $\mathbf{B}$ follows, so only the case $\Lambda \neq 0$ needs further investigation.

Dotting $\Omega$ from the right on Eq. (97), we have

$$
\mathrm{B} \cdot \Omega=\nabla \Omega \cdot \Omega=\frac{1}{2} \nabla\left(\Omega^{2}\right)=-\frac{1}{4} \nabla \Lambda=0,
$$

revealing $\Omega$ as an eigenvector of $B$ with eigenvalue zero. Since $\operatorname{Tr} B=0$, the other eigenvalues must be equal in magnitude but opposite in sign, allowing $B$ to be written

$$
\mathbf{B}=b(\mathbf{v} \mathbf{v}-\mathbf{w w})
$$

where $\mathbf{v}$ and $\mathbf{w}$ are the other two orthonormal eigenvectors. The scalar $b$ may be expressed in terms of the only non-zero invariant of $B$ as follows:

$$
\mathbf{B} \cdot \mathbf{B}=b^{2}(\mathbf{v v}+\mathbf{w w})=b^{2}\left(1-\frac{1}{\Omega^{2}} \Omega \Omega\right),
$$

giving

$$
\operatorname{Tr} \mathbf{B} \cdot \mathbf{B}=\mathbf{B}: \mathbf{B}=2 b^{2},
$$

and so, of course, $\dot{b}=0$. Note that all the eigenvectors of $B$ are body-fixed, and they may therefore be adopted for an intrinsic orthonormal basis triad. This we now do, for the remainder of this section.

It is convenient to introduce a symbol $C$ for the traceless dyadic $\Omega \times B$. The antisymmetric part of $\Omega \times B$ is found using Eqs. (A-6) and $(\hat{A}-13)$ to be

$$
-\frac{1}{2}[(\Omega \times B) \dot{\times} I] \times I=\frac{1}{2}[(\operatorname{Tr} B) \Omega-\Omega \cdot B] \times I,
$$

and since this vanishes, $C$ is symmetric. Thus, we put

$$
\mathbf{C}=\Omega \times \mathbf{B}=(\Omega \times B)^{T}=-\mathrm{B} \times \Omega
$$

with

$$
\Omega \cdot C=C \cdot \Omega=\operatorname{Tr} C=0 .
$$

Using $C$ and Eqs. (94), (96), and (98), the Bianchi identities in Eqs. (70) to (73) of Section II-D may now be written

$$
\boldsymbol{\nabla} \times \mathbf{A}=\mathbf{C}, \quad \boldsymbol{\nabla} \cdot \mathbf{A}=\mathbf{0}
$$

$$
\nabla \times B=-\frac{1}{2} \Lambda \Omega \times I, \quad \nabla \cdot B=\Lambda \Omega .
$$


We complete this set by evaluating $\nabla \times \mathrm{C}$. Using the identity equation (A-25), Eq. (97), and the above value for $\nabla \times \mathrm{B}$, we have

$$
\begin{aligned}
\boldsymbol{\nabla} \times \mathbf{C} & =-\nabla \times(\mathbf{B} \times \Omega)=-(\nabla \times \mathbf{B}) \times \Omega+\mathbf{B} \times(\nabla \Omega) \\
& =\frac{\Lambda}{2}\left[\Omega \Omega-\left(\Omega^{2}\right) \mathbf{l}\right]+2 \mathbf{B} \cdot \mathbf{B}-(\mathbf{B}: \mathbf{B}) \mathbf{I}, \quad(107)
\end{aligned}
$$

where, in the last equality, we have also employed the expansion equation (A-4) for $\mathbf{B}_{\mathbf{X}}^{\mathbf{B}} \mathbf{B}$. As shown in Eq. (A-28), $\operatorname{Tr}(\boldsymbol{\nabla} \times \mathbf{C})=0$ for symmetric $C$ so that, by tracing Eq. (107) and using Eq. (94), we find

$$
0=\frac{1}{2} \Lambda^{2}-B: B
$$

or

$$
b^{2}=\left(\frac{\Lambda}{2}\right)^{2}
$$

Using this result and Eq. (100), we have

$$
\boldsymbol{\nabla} \times \mathbf{C}=\frac{\Lambda}{2}\left[3 \Omega \Omega-\left(\Omega^{2}\right) \mathbf{I}\right], \quad \boldsymbol{\nabla} \cdot \mathbf{C}=0 .
$$

The following expressions, which are needed below, are easily obtained by combining previous equations:

$$
\begin{aligned}
& C \cdot B=\Omega \times B \cdot B=\left(\frac{\Lambda}{2}\right)^{2} \Omega \times I, \\
& C_{\times}^{\times} C=\frac{1}{2} \Lambda^{2} \Omega \Omega, \\
& C_{\times}^{\times} B=0 .
\end{aligned}
$$

We are now able to determine the anholonomic affinity of the quotient space in terms of intrinsic quantities. Applying to $\Omega$ the general equation (49) for covariant differentiation, we have

$$
\nabla \Omega=\mathrm{D} \Omega-\mathbf{N} \times \boldsymbol{\Omega}=\mathbf{B} .
$$

Since $\Omega$ is an eigenvector of $B$ and has constant magnitude, its components in the adopted basis triad are constant; by definition, therefore, $D \Omega=0$. Crossing $\Omega$ from the right in Eq. (114) and solving for $\mathbf{N}$ leads to

$$
\frac{\Lambda}{2} \mathbf{N}=-B \times \Omega-\mathbf{N} \cdot \Omega \Omega,
$$

which expresses $\mathbf{N}$ in intrinsic quantities, except for the vector, $\mathbf{H} \equiv \mathbf{N} \cdot \boldsymbol{\Omega}$. Rewriting, we have

$$
\frac{\Lambda}{2} \mathbf{N}=\mathbf{C}-\mathbf{H} \boldsymbol{\Omega}
$$

with the corollaries

$$
\frac{\Lambda}{2} \mathbf{N}^{T}=\mathbf{C}-\mathbf{\Omega H}
$$

and

$$
\frac{\Lambda}{2} \operatorname{Tr} \mathbf{N}=-\mathbf{H} \cdot \mathbf{\Omega}
$$

The components of $B$ in the adopted triad are also constant, so that $\mathbf{D} \times \mathbf{B}=0$. Writing the covariant curl of $\mathbf{B}$ according to Eq. (50) and substituting for the affinity from Eqs. (116) to (118), we have

$$
\begin{aligned}
\frac{\Lambda}{2} \nabla \times B= & -(\Omega \cdot \mathbf{H}) B-C \cdot B \\
& +\Omega H \cdot B-C_{\times}^{\times} B+\Omega H_{\times}^{\times} B
\end{aligned}
$$

From Eqs. (106), (111), and (113), we see that

$$
\frac{\Lambda}{2} \nabla \times B=-C \cdot B, \quad C_{\times}^{\times} B=0,
$$

while from the expansion formula (A-4)

$$
\boldsymbol{\Omega} \mathbf{H}_{\mathbf{X}}^{\mathbf{X}} \mathbf{B}=\boldsymbol{\Omega} \mathbf{H} \cdot \mathbf{B}-(\boldsymbol{\Omega} \cdot \mathbf{H}) \mathbf{B},
$$

so that, removing a factor of 2, Eq. (119) results in

$$
\Omega \mathbf{H} \cdot \mathbf{B}-(\boldsymbol{\Omega} \cdot \mathbf{H}) \mathbf{B}=\mathbf{0} .
$$

Dotting $\Omega$ from the left in this equation gives

$$
\left(\Omega^{2}\right) \mathbf{H} \cdot \mathbf{B}=0
$$

We reject the possibility $\left(\Omega^{2}\right)=-1 / 2 \Lambda=0$, and settle for

$$
\mathbf{H} \cdot \mathbf{B}=0 \text {. }
$$

But Eq. (120) then also requires $(\Omega-\mathbf{H}) B=0$, and rejecting $B=0$ [since it entails also $\Lambda=0$, by Eq. (109)], we take $\boldsymbol{\Omega} \cdot \mathbf{H}=0$, stating that $\boldsymbol{\Omega}$ and $\mathbf{H}$ are orthogonal. However, with the structure of B given in Eq. (99), 
Eq. (122) demands that $\mathbf{H}$ be collinear with $\Omega$, and we are finally forced to accept that

$$
\mathbf{H}=0 .
$$

We now have $\mathbf{N}$ explicitly,

$$
\frac{\Lambda}{2} \mathbf{N}=\frac{\Lambda}{2} \mathbf{N}^{T}=\mathbf{C}
$$

The coup-de-grâce is accomplished when this value for $\mathbf{N}$ and the value of $\mathbf{E}$ determined from the Gauss equation (61),

$$
\mathbf{E}=-2 \Omega \Omega-\left(\Omega^{2}\right) \mathbf{I},
$$

are substituted into Eq. (51), which defines E;

$$
\boldsymbol{\nabla} \times \mathbf{N}=-\frac{1}{2} \mathbf{N}_{\times}^{T} \times \mathbf{N}+\mathbf{E} .
$$

Multiplying through by $1 / 2 \Lambda^{2}$, we obtain

$$
\Lambda \nabla \times C=-C_{\times}^{\times} C-\frac{1}{2} \Lambda^{2}\left[2 \Omega \Omega+\left(\Omega^{2}\right) I\right],
$$

and using Eqs. (110) and (112), this becomes

$$
3 \Lambda^{2} \Omega \Omega=0 .
$$

There is now no escape from the string of consequences

$$
\Lambda=\mathbf{\Omega}=\mathbf{A}=\mathbf{B}=\mathbf{E}=0,
$$

which reduces this class of motions to uniform translation in flat space-time. As a result, in the succeeding sections of this report, we may always assume $\mathbf{a} \neq 0$.

\section{IRROTATIONAL RIGID FRAMES}

As in the flat and constant curvature space-times of Section III, the class of irrotational rigid motions proves to be the most interesting even for arbitrary exterior gravitational fields. Although we have not quite been able to carry through a complete proof of the HerglotzNoether theorem, it will be shown in Section VI that the motion of the rotating rigid frame in any exterior field is totally determined by initial conditions on a spacelike hypersurface. Only among the irrotational frames can we find any motions allowing arbitrary functions of time, or in other words, degrees of freedom. We shall concentrate here on finding all such nonisometric normal rigid motions which may exist in an arbitrary
Einstein space. The variety of exterior metrics admitting such motions will also be obtained; they turn out to be quite special, falling into a well-known class of Einstein spaces.

The equations for these motions are, of course, obtained by putting $\Omega=0$ throughout Section II-D. We note, first of all, that the potential equation (69) for $\Omega$ becomes $\boldsymbol{\nabla} \times \mathbf{A}=0$, and accordingly we may select a gauge such that $\mathbf{A}=0$. This simply corresponds to using a time coordinate whose surfaces coincide with the normal hypersurfaces of the congruence. Next we find that Eq. (66) reduces to $B=0$, and the gravitational field 
is thus described by $\mathbf{A}$ alone. Here we insist that $\mathbf{A} \neq \mathbf{0}$, since the manifold would otherwise reduce to a spacetime of constant curvature. This structure of the field dyadics can only be obtained in Type I and D Einstein spaces in the Petrov-Pirani-Sachs classification scheme, as pointed out previously by Pirani and Williams (Ref. 8).

Turning to the acceleration equations, we expect from Eq. (64), which reads $\nabla \times \mathbf{a}=0$, that a will have a scalar potential. With the choice of gauge already made, Eq. (65) becomes simply

$$
\phi \mathbf{a}=-\boldsymbol{\nabla} \phi,
$$

so that the potential can be taken as $-\ln \phi$. The dyadic differential equation (62) for a reduces to

$$
\boldsymbol{\nabla} \mathbf{a}=-\mathbf{a} \mathbf{a}+\mathbf{A}-\frac{1}{3} \Lambda \mathbf{l}
$$

and the Bianchi identity for $\mathbf{A}$ becomes

$$
\boldsymbol{\nabla} \times \mathbf{A}=-\mathbf{a} \times \mathbf{A}+\mathbf{A} \times \mathbf{a},
$$

with the antisymmetric part

$$
\boldsymbol{\nabla} \cdot \mathbf{A}=\mathbf{0} .
$$

Finally, the Gauss equation (61) is

$$
\mathbf{E}=-\mathbf{A}+\frac{1}{3} \Lambda \mathbf{l}
$$

and since $\dot{\boldsymbol{E}}=0$, we find from this (or from the other Bianchi identity) that $\dot{\mathbf{A}}=\mathbf{0}$ as well. The only intrinsic quantities for which time dependence is permissible then are the acceleration vector a and its potential $\phi$. Since we are not presently interested in isometric motions, we assume henceforth that $\dot{\mathbf{a}} \neq 0$, and attempt to construct solutions obeying this constraint. In order to demonstrate that the solutions obtained exhaust all possibilities, we shall exhibit the construction in some detail.

First the algebraic properties of the dyadic $A$ will be examined. Using the fact that $\dot{A}=0$, we time differentiate the Bianchi identity equation (131) and find

$$
(\boldsymbol{\nabla} \times \mathbf{A})^{\cdot}=-\dot{\mathbf{a}} \times \mathbf{A}+\mathbf{A} \times \dot{\mathbf{a}}=0,
$$

where the commutation law equation (54) justifies equating this to zero. This equation states that the symmetric part of the dyadic $\dot{\mathbf{a}} \times \mathbf{A}$ vanishes, so that we may write (Appendix A)

$$
\dot{\mathbf{a}} \times \mathbf{A}=-\mathbf{h} \times \mathbf{I},
$$

where $h$ is the vector defined by

$$
\mathbf{h}=\frac{1}{2}(\dot{\mathbf{a}} \times \mathbf{A}) \dot{x} \mathbf{l}
$$

Using the identity (A-13) and $\operatorname{Tr} A=0$, we find

$$
\mathbf{h}=\frac{1}{2} \dot{\mathbf{a}} \cdot \mathbf{A} .
$$

If we dot a from the left in Eq. (135), however, we get

$$
\mathbf{h} \times \dot{\mathbf{a}}=\mathbf{0} \text {, }
$$

so we must be able to write

$$
\mathbf{h}=\boldsymbol{\alpha}^{3} \dot{\mathbf{a}},
$$

where the proportionality factor is written as $\alpha^{3}$ simply to avoid fractional exponents later. Combining Eqs. (137) and (138), we have

$$
\dot{\mathbf{a}} \cdot \mathbf{A}=2 \alpha^{3} \dot{\mathbf{a}},
$$

which exhibits $\dot{\mathbf{a}}$ as an eigenvector of $\mathbf{A}$ with eigenvalue $2 \alpha^{3}$. If we now cross $\dot{a}$ from the left on Eq. (135), we obtain with the use of identities (A-8) and (A-9)

$$
\dot{\mathbf{a}} \mathbf{a} \cdot \mathbf{A}-|\dot{\mathbf{a}}|^{2} \mathbf{A}=-\mathbf{h} \dot{\mathbf{a}}+(\dot{\mathbf{a}} \cdot \mathbf{h}) \mathbf{l},
$$

and substituting from Eqs. (138) and (139), we may solve for $A$ as

$$
\mathbf{A}=\alpha^{3}\left[3 \frac{\dot{\mathbf{a}} \dot{\mathbf{a}}}{|\dot{\mathbf{a}}|^{2}}-\mathbf{I}\right]
$$

It will be convenient to define a unit vector, $\mathbf{u}=\dot{\mathbf{a}} /|\dot{\mathbf{a}}|$, and so to write Eq. (140) as

$$
A=\alpha^{3}[3 \mathbf{u u}-1] .
$$

It is easily verified that $A: A=6 \alpha^{6}$, and the time derivative of this shows $\dot{\alpha}=0$. Now time differentiating Eq. (141) and using the fact that $u$ is a unit vector, we find $\dot{\mathbf{u}}=0$, so that $\mathbf{u}$ is a body-fixed unit vector. So far then, we have obtained an explicit form for the field dyadic in terms of the time derivative of the acceleration vector $\dot{\mathbf{a}}$; we have determined that this vector is an 
eigenvector of $\mathbf{A}$ and that its direction is fixed in the rigid frame (only its magnitude can vary with time).

Clearly, the vector $\mathbf{u}$ should be adopted as one of the intrinsic triad basis vectors; we now find two more to complete the triad. Let $\mathbf{a}_{\perp}$ be the component of the acceleration perpendicular to $\dot{a}$, so that

$$
\mathbf{a}_{\perp} \equiv \mathbf{a}-(\mathbf{a} \cdot \mathbf{u}) \mathbf{u}
$$

We verify that this is a time-independent, body-fixed vector by

$$
\dot{\mathbf{a}}_{\perp}=\dot{\mathbf{a}}-\langle\dot{\mathbf{a}} \cdot \mathbf{u}) \mathbf{u}=|\dot{\mathbf{a}}| \mathbf{u}-|\dot{\mathbf{a}}| \mathbf{u}=0,
$$

and so we can use it to define a second intrinsic triad vector $\mathbf{v}$ by writing

$$
\mathbf{a}_{\perp}=\left|\mathbf{a}_{\perp}\right| \mathbf{v}=(\mathbf{a} \cdot \mathbf{v}) \mathbf{v}
$$

where

$v^{2}=1, \quad \mathbf{u} \cdot \mathbf{v}=0, \quad \dot{\mathbf{v}}=0, \quad(\mathbf{a} \cdot \mathbf{v})^{\bullet}=0$.

The third triad vector $\mathbf{w}$ is defined simply by $\mathbf{w} \equiv \mathbf{u} \times \mathbf{v}$, and, of course, $\mathbf{a} \cdot \mathbf{w}=\dot{\mathbf{a}} \cdot \mathbf{w}=\mathbf{0}$. This construction will fail if $\mathbf{a}$ and $\dot{\mathbf{a}}$ are collinear; but for now we assume this is not the case. We shall return to consider the collinear case later; it is fairly easily obtained as a limit of the more general solution.

Having adopted a basis triad, we proceed to investigate its differential properties. From its definition, we may write

$$
\nabla \mathbf{u}=\nabla(\dot{\mathbf{a}} /|\dot{\mathbf{a}}|)-\frac{1}{|\dot{\mathbf{a}}|}[\nabla(\dot{\mathbf{a}})-(\nabla|\dot{\mathbf{a}}|) \mathbf{u}]
$$

The right-hand side of this equation is evaluated by combining the commutation relation equation (54) and the time derivative of Eq. (130). We have

$$
\nabla(\dot{\mathbf{a}})=(\nabla \mathbf{a})^{\bullet}-\mathbf{a} \dot{\mathbf{a}}=-\dot{\mathbf{a}} \mathbf{a}-2 \mathbf{a} \dot{\mathbf{a}}=-|\dot{\mathbf{a}}|[\mathbf{u} \mathbf{a}+2 \mathbf{a u}],
$$

and from this

$$
\nabla|\dot{\mathbf{a}}|=\frac{1}{|\dot{\mathbf{a}}|} \nabla(\dot{\mathbf{a}}) \cdot \dot{\mathbf{a}}=\nabla(\dot{\mathbf{a}}) \cdot \mathbf{u}=-|\dot{\mathbf{a}}|[(\mathbf{a} \cdot \mathbf{u}) \mathbf{u}+2 \mathbf{a}]
$$

Substituting in Eq. (144), we find

$$
\boldsymbol{\nabla} \mathbf{u}=-\mathbf{u}[\mathbf{a}-(\mathbf{a} \cdot \mathbf{u}) \mathbf{u}]=-\mathbf{u} \mathbf{a}_{\perp}=-(\mathbf{a} \cdot \mathbf{v}) \mathbf{u v},
$$

and the corollaries

$$
\boldsymbol{\nabla} \times \mathbf{u}=(\boldsymbol{\nabla} \mathbf{u}) \dot{\dot{x}} \mathbf{l}=-(\mathbf{a} \cdot \mathbf{v} \mathbf{w}, \quad \boldsymbol{\nabla} \cdot \mathbf{u}=(\boldsymbol{\nabla} \mathbf{u}): \mathbf{l}=\mathbf{0} .
$$

In like manner, we evaluate $\nabla \mathbf{v}=\boldsymbol{\nabla}\left(\mathbf{a}_{\perp} /\left|\mathbf{a}_{\ell}\right|\right)$ by first calculating

$$
\begin{aligned}
\boldsymbol{\nabla} \mathbf{a}_{\perp}= & \boldsymbol{\nabla}[\mathbf{a}-(\mathbf{a} \cdot \mathbf{u}) \mathbf{u}]=\boldsymbol{\nabla} \mathbf{a}-\boldsymbol{\nabla} \mathbf{a} \cdot \mathbf{u} \mathbf{u} \\
& -\boldsymbol{\nabla} \mathbf{u} \cdot \mathbf{a u}-(\mathbf{a} \cdot \mathbf{u}) \boldsymbol{\nabla} \mathbf{u}
\end{aligned}
$$

and substituting from Eqs. (130), (141), and (145). The result is

$$
\boldsymbol{\nabla} \mathbf{v}=(\mathbf{a} \cdot \mathbf{v}) \mathbf{u u}-\frac{\left(\alpha^{3}+1 / 3 \Lambda\right)}{(\mathbf{a} \cdot \mathbf{v})} \mathbf{w w},
$$

and

$\boldsymbol{\nabla} \times \mathbf{v}=0, \quad \boldsymbol{\nabla} \cdot \mathbf{v}=\frac{1}{(\mathbf{a} \cdot \mathbf{v})}\left[(\mathbf{a} \cdot \mathbf{v})^{2}-\alpha^{3}-1 / 3 \Lambda\right]$.

Finally, $\nabla \mathbf{w}$ is now easily obtained from

$$
\boldsymbol{\nabla} \mathbf{w}=\boldsymbol{\nabla}(\mathbf{u} \times \mathbf{v})=\boldsymbol{\nabla} \mathbf{u} \times \mathbf{v}-\boldsymbol{\nabla} \mathbf{v} \times \mathbf{u},
$$

giving

$$
\boldsymbol{\nabla} \mathbf{w}=\frac{\left(\alpha^{3}+1 / 3 \Lambda\right)}{(\mathbf{a} \cdot \mathbf{v})} \mathbf{w v},
$$

with

$$
\boldsymbol{\nabla} \times \mathbf{w}=-\frac{\left(\alpha^{3}+1 / 3 \Lambda\right)}{(a \cdot v)} \mathbf{u}, \quad \boldsymbol{\nabla} \cdot \mathbf{w}=0 .
$$

The geometrical content of these equations may be summarized by the statements that $\mathbf{v}$ is a $\mathcal{2}$-space normal, geodesic vector of the quotient space, while $u$ and $w$ are 2-space normal, Killing vectors of the quotient space.

We are now prepared to calculate the gradients of all the independent intrinsic scalars which can be formed; viz., $\phi, \mathbf{a} \cdot \mathbf{u}, \mathbf{a} \cdot \mathbf{v}$, and $\alpha$. All other scalars are algebraic functions or derivatives of these. The potential equation (129) gives $\nabla_{\phi}$ directly,

$$
\boldsymbol{\nabla}_{\boldsymbol{\phi}}=-\phi[(\mathbf{a} \cdot \mathbf{u}) \mathbf{u}+(\mathbf{a} \cdot \mathbf{v}) \mathbf{v}] .
$$

The next two are evaluated as

$$
\begin{aligned}
\boldsymbol{\nabla}(\mathbf{a} \cdot \mathbf{u})= & \boldsymbol{\nabla} \mathbf{a} \cdot \mathbf{u}+\boldsymbol{\nabla} \mathbf{u} \cdot \mathbf{a}=-\left(a^{2}-2 \alpha^{3}+1 / 3 \Lambda\right) \mathbf{u} \\
& -(\mathbf{a} \cdot \mathbf{u})(\mathbf{a} \cdot \mathbf{v}) \mathbf{v}
\end{aligned}
$$


and similarly

$$
\boldsymbol{\nabla}(\mathbf{a} \cdot \mathbf{v})=-\left[(\mathbf{a} \cdot \mathbf{v})^{2}+\alpha^{3}+1 / 3 \Lambda\right] \mathbf{v} .
$$

The gradient of $\alpha$ is most easily derived by substituting the explicit form for A given in Eq. (141) into the field equation (132). Using the identity (A-18) for chain differentiation, we have

$$
\boldsymbol{\nabla} \cdot \mathbf{A}=\boldsymbol{\nabla}\left(\boldsymbol{\alpha}^{3}\right) \cdot[3 \mathbf{u u}-1]+3 \alpha^{3}[(\nabla \cdot \mathbf{u}) \mathbf{u}+\mathbf{u} \cdot \nabla \mathbf{u}]=0
$$

By rearranging and substituting from Eqs. (145) and (146), this becomes

$$
\alpha^{2} \nabla \alpha-3 \alpha^{2}(\mathbf{u} \cdot \nabla \alpha) \mathbf{u}+\alpha^{3}(\mathbf{a} \cdot \mathbf{v}) \mathbf{v}=0 .
$$

Dotting $\mathbf{u}$ through this equation we find $\mathbf{u} \cdot \nabla_{\alpha}=0$, so that finally

$$
\nabla \alpha=-\alpha(\mathbf{a} \cdot \mathbf{v}) \mathbf{v} .
$$

Comparison of Eqs. (153) and (154) now reveals that an integral exists; using these equations, it is found that

$$
\nabla\left[\frac{(\mathbf{a} \cdot \mathbf{v})^{2}-2 \alpha^{3}+1 / 3 \Lambda}{\alpha^{2}}\right]=0 .
$$

By integrating and solving for $(\mathbf{a} \cdot \mathbf{v})^{2}$, this may be written as

$$
(\mathbf{a} \cdot \mathbf{v})^{2}={ }_{\varepsilon} k^{2} \alpha^{2}+2 \alpha^{3}-1 / 3 \Lambda,
$$

where we use an indicator $\varepsilon(\varepsilon=+1,-1)$ to account for sign and may restrict the integration constant $k$ so that $k \supseteq 0$. We have, of course, $\nabla k=0$, but since all other quantities in Eq. (156) have already been shown to be time independent, it follows that $\dot{k}=0$ as well.

It is not quite so obvious that the set of equations (151) to (154) leads also to another integral. Using them together with the integral equation (156), we may verify, however, that

$$
\nabla\left[\frac{(\mathbf{a} \cdot \mathbf{u})^{2}+\varepsilon k^{2} \alpha^{2}}{\phi^{2}}\right]=0
$$

and so obtain

$$
(\mathbf{a} \cdot \mathbf{u})^{2}+\varepsilon k^{2} \alpha^{2}=\varepsilon^{\prime}\left(k^{\prime}\right)^{2} \phi^{2},
$$

where $\varepsilon^{\prime}$ is another sign indicator $\left(\varepsilon^{\prime}=+1,-1\right)$ and $k^{\prime} \supseteq 0$ with $\nabla k^{\prime}=0$. Here $k^{\prime}$ might be a function of time; since it enters only as a factor of the potential $\phi$, such time dependence may, however, be absorbed by a special gauge transformation [Eq. (21)], as discussed in Section II. The integral now becomes simply

$$
(\mathbf{a} \cdot \mathbf{u})^{2}=\varepsilon^{\prime} \phi^{2}-\varepsilon k^{2} \alpha^{2}
$$

We note that this equation forbids the combination: $\varepsilon^{\prime}=-1, \varepsilon=+1$; all other combinations are in general allowed. Adding Eqs. (156) and (159), we obtain the square of the acceleration vector

$$
a^{2}=\varepsilon^{\prime} \phi^{2}+2 \alpha^{3}-1 / 3 \Lambda .
$$

Physically reasonable space-times are usually associated with $\Lambda \supseteq 0$; for these cases, we see from Eqs. (156) and (160) that, if $\alpha$ is negative, the only allowed combination of signs is $\varepsilon^{\prime}=\varepsilon=+1$.

At this point, all the essential intrinsic relations have been derived; it remains only to adopt a system of comoving coordinates and obtain the corresponding metrics of the quotient space and of space-time. As mentioned before, all three of the adopted triad vectors fortunately turn out to be 2-space normal. We may, consequently, expect to be able to introduce an orthogonal coordinate system in the quotient space.

Two of the intrinsic scalars, $(\mathbf{a} \cdot \mathbf{v})$ and $\alpha$, offer themselves as candidates for intrinsic co-moving coordinates, since we have verified that they are time independent. Actually, since these are related by Eq. (156), only one independent intrinsic coordinate is available. Any function of $\alpha$ and $(a \cdot v)$ might be selected, but a convenient choice proves to be $-(k \alpha)^{-1}$. According to Eq. (154), the triad vector $\mathrm{v}$ is normal to the 2-spaces $\alpha=$ constant, so we adopt the symbol $y$ for this coordinate and solve for the scalars in terms of it:

$$
\begin{gathered}
\alpha=-\frac{1}{k y}, \\
(\mathbf{a} \cdot \mathbf{v})^{2}=\frac{\varepsilon}{y^{2}}-\frac{2}{(k y)^{3}}-1 / 3 \Lambda .
\end{gathered}
$$

By differentiating Eq. (161), we have

$$
\nabla \alpha=\frac{1}{k y^{2}} \nabla y,
$$

so that Eq. (154) may be transformed to

$$
\mathbf{e}^{2} \equiv \nabla y=y(\mathrm{a} \cdot \mathbf{v}) \mathbf{v},
$$


where we have introduced the natural basis vector $\mathbf{e}^{2}$ in accordance with the discussion of Section II-B.

Intrinsic co-moving potentials for the other triad vectors are not available; this is connected with the fact that $\mathbf{u}$ and $\mathbf{w}$ were found to be Killing vectors, implying spatial symmetry of the problem in these directions. We know, however, that the space congruences generated by $\mathbf{u}$ and w are each body-fixed and 2-space normal, and of course orthogonal to each other. Accordingly, we may introduce orthogonal, body-fixed (co-moving) coordinates, say $\bar{x}$ and $\bar{z}$, respectively, by any arbitrary time-independent labeling of the 2-surfaces normal to these congruences. For the gradient vectors, we may write then

$$
\begin{aligned}
& \nabla \bar{x}=\frac{1}{y} \xi(\bar{x}, y, \bar{z}) \mathbf{u}, \\
& \nabla \bar{z}=\frac{1}{y(\mathbf{a} \cdot \mathbf{v})} \zeta(\bar{x}, y, \bar{z}) \mathbf{w} .
\end{aligned}
$$

The arbitrary scale factors $\xi$ and $\zeta$ allow for the fact that the magnitudes of the gradient vectors on the left are as yet unknown, while the factors $1 / y$ and $1 / y(a \cdot v)$ have been written separately for convenience.

The integrability conditions for these equations are simply

$$
\nabla \times \nabla \bar{x}=\nabla \times \nabla(\bar{z})=0,
$$

and taking the curls of the right-hand sides, we easily find they reduce to

$$
\begin{aligned}
\boldsymbol{\nabla} \xi & =(\mathbf{u} \cdot \boldsymbol{\nabla} \xi) \mathbf{u}, \\
\boldsymbol{\nabla} \zeta & =(\mathbf{w} \cdot \mathbf{\nabla} \zeta) \mathbf{w} .
\end{aligned}
$$

But these equations demand that $\xi(\bar{x})$ be a function of $\bar{x}$ only, and likewise $\zeta(\bar{z})$ a function of $\bar{z}$ only. It is permissible then to define a new pair of coordinates, $x$ and $z$, by the equations

$$
\frac{d x}{d \bar{x}}=\frac{1}{\xi(\bar{x})}, \quad \frac{d z}{d \bar{z}}=\frac{1}{\zeta(\bar{z})} .
$$

Introducing the natural basis vectors $\mathbf{e}^{1}$ and $\mathbf{e}^{3}$, we now have

$$
\begin{aligned}
& \mathbf{e}^{1} \equiv \nabla x=\frac{d x}{d \bar{x}} \nabla \bar{x}=\frac{1}{y} \mathbf{u} \\
& \mathbf{e}^{3} \equiv \nabla z=\frac{d z}{d \bar{z}} \nabla \bar{z}=\frac{1}{y(\mathbf{a} \cdot \mathbf{v})} \mathbf{w}
\end{aligned}
$$

The dual natural triad is clearly

$$
\mathbf{e}_{1}=y \mathbf{u}, \quad \mathbf{e}_{2}=\frac{1}{y(\mathbf{a} \cdot \mathbf{v})} \mathbf{v}, \quad \mathbf{e}_{3}=y(\mathbf{a} \cdot \mathbf{v}) \mathbf{w},
$$

and the non-zero covariant metric coefficients of the quotient space are consequently given by

$$
\begin{aligned}
& h_{11}=\mathbf{e}_{1} \cdot \mathbf{e}_{1}=y^{2}, \\
& h_{22}=\mathbf{e}_{2} \cdot \mathbf{e}_{2}=\frac{1}{y^{2}(\mathbf{a} \cdot \mathbf{v})^{2}}=\left[\varepsilon-\frac{2}{k^{3} y}-\frac{1}{3} \Lambda y^{2}\right]^{-1}, \\
& h_{33}=\mathbf{e}_{3} \cdot \mathbf{e}_{3}=y^{2}(\mathbf{a} \cdot \mathbf{v})^{2}=\left[\varepsilon-\frac{2}{k^{3} y}-\frac{1}{3} \Lambda y^{2}\right] .
\end{aligned}
$$

Since the constant $k$ occurs here only as $k^{-3}$, we shall henceforth write $m$, where $m=k^{-3}$ is a positive constant. The square of the interval in the quotient space is given then in this set of co-moving coordinates by

$$
\begin{aligned}
d l^{*}= & y^{2} d x^{2}+\left(\varepsilon-\frac{2 m}{y}-\frac{1}{3} \Lambda y^{2}\right)^{-1} d y^{2} \\
& +\left(\varepsilon-\frac{2 m}{y}-\frac{1}{3} \Lambda y^{2}\right) d z^{2}
\end{aligned}
$$

Note that the metric coefficients are independent of $x$ and $z$; this expresses the symmetry properties we expected from the existence of two Killing vectors, $\mathbf{u}$ and $\mathbf{w}$.

To write the space-time metric in the form of Eq. (24) of Section II, we need only obtain an explicit expression for the potential $\phi$, since we are employing the gauge $\mathbf{A}=0$. To find $\phi$, we write the obvious equality

$$
y_{\phi}[\mathbf{a}-(\mathbf{a} \cdot \mathbf{u}) \mathbf{u}-(\mathbf{a} \cdot \mathbf{v}) \mathbf{v}]=\mathbf{0}
$$

and insert a from Eq. (129), $(\mathbf{a} \cdot \mathbf{u})$ from $(154)$, $\mathbf{u}$ from $(165)$, and $(a \cdot v) v$ from Eq. (164). The result of all these substitutions is

$$
\nabla(y \phi) \pm(y \phi)\left[\mathbf{\varepsilon}^{\prime}(y \phi)^{2}-\varepsilon\right]^{1 / 2} \nabla x=0 .
$$

Consider first the case $\varepsilon^{\prime}=\varepsilon=1$; from Eq. (159), we find $(y \phi)^{2} \supseteq 1$, so that an appropriate substitution will be

$$
y_{\phi}=\csc \theta \text {. }
$$

With this substitution, Eq. (170) becomes

$$
\nabla \theta= \pm \nabla x,
$$


and so

$$
\theta= \pm[x+f(t)]
$$

where $\nabla f=0$, but $f$ is allowed to be any function of the $t$ coordinate, since again we have chosen the gauge $A \equiv \nabla t=0$. Solving for $1 / \phi$, we have

$$
\frac{1}{\phi}= \pm y \sin [x+f(t)],
$$

and according to Eq. (24), the space-time interval in these co-moving coordinates has the form

$$
\text { I. } \begin{aligned}
\varepsilon=\varepsilon^{\prime}=1, & (y \phi)^{2} \geqslant 1 \\
d s^{2}= & y^{2}\left\{-\sin ^{2}[x+f(t)] d t^{2}+d x^{2}\right\} \\
& +\left(1-\frac{2 m}{y}-\frac{1}{3} \Lambda y^{2}\right)^{-1} d y^{2} \\
& +\left(1-\frac{2 m}{y}-\frac{1}{3} \Lambda y^{2}\right) d z^{2}
\end{aligned}
$$

Exactly analogous integrations of Eq. (170) for the remaining two permitted combinations of signs lead to

II. $\varepsilon=-1, \varepsilon^{\prime}=1, \quad 0 \leq(y \phi)^{2} \leq \infty$

$$
\begin{aligned}
d s^{2}= & y^{2}\left(-\sinh ^{2}[x+f(t)] d t^{2}+d x^{2}\right\} \\
& +\left(-1-\frac{2 m}{y}-\frac{1}{3} \Lambda y^{2}\right)^{-1} d y^{2} \\
& +\left(-1-\frac{2 m}{y}-\frac{1}{3} \Lambda y^{2}\right) d z^{2}
\end{aligned}
$$

and

$$
\text { III. } \begin{aligned}
\varepsilon= & \varepsilon^{\prime}=-1, \quad(y \phi)^{2} \leq 1 \\
d s^{\prime \prime}= & y^{2}\left\{-\cosh ^{2}[x+f(t)] d t^{2}+d x^{2}\right\} \\
& +\left(-1-\frac{2 m}{y}-\frac{1}{3} \Lambda y^{2}\right)^{-1} d y^{2} \\
& +\left(-1-\frac{2 m}{y}-\frac{1}{3} \Lambda y^{2}\right) d z^{2}
\end{aligned}
$$

These metrics have meaning only when the coefficients of $d y^{2}$ and $d z^{2}$ are positive; this is also clear intrinsically from Eq. (162), which shows that

$$
y^{2}(a \cdot v)^{2}=\varepsilon-\frac{2 m}{y}-\frac{1}{3} \Lambda y^{2} .
$$

In metrics II and III, for example, we must at least have $-2 m<y<0$.

Returning now to the special case left behind, which was characterized precisely by $\mathbf{a}_{\perp}=\mathbf{a} \cdot \mathbf{v}=0$, we see that it appears as a singular limit. After investigating some of its properties, we can obtain the metrics for this situation. Reference to Eq. (145) shows that in this case $\nabla \mathbf{u}=0$, so that $\mathbf{u}$ is a covariant constant vector in the quotient space. The integrability condition for this can be found using Eq. (60) of Section II-D;

$\mathbf{E} \times \mathbf{u}=\left(-\mathbf{A}+\frac{1}{3} \Lambda \mathbf{l}\right) \times \mathbf{u}=\left(\alpha^{3}+\frac{1}{3} \Lambda\right) \mathbf{u} \times \mathbf{I}=0$,

where we have used Eqs. (133) and (141). Thus, we require

$$
\alpha^{3}=-\frac{1}{(k y)^{3}}=-\frac{1}{3} \Lambda .
$$

The dyadics $\mathbf{A}$ and $\mathbf{E}$ become then

$$
\begin{aligned}
& \mathbf{A}=-\frac{1}{3} \Lambda(3 \mathbf{u u}-\mathbf{I}), \\
& \mathbf{E}=\Lambda \mathbf{u u},
\end{aligned}
$$

and we see that a nontrivial solution exists here only for $\Delta \neq 0$. Following through a direct integration of the dyadic equations leads in one case to the space-time metric

$$
\begin{aligned}
& \text { IV. } a_{1}=0, \quad \Lambda>0 \\
& \qquad \begin{aligned}
d s^{2}= & \frac{1}{\Lambda}\left\{-\sin ^{2}[x+f(t)] d t^{2}+d x^{2}+d \theta^{2}\right. \\
& \left.+\sin ^{2} \theta d \phi^{2}\right\}
\end{aligned}
\end{aligned}
$$

However, this last may be more simply obtained as a limit at the coordinate singularity of metric I, Eq. (174). Note that the $y$ coordinate becomes constant, $y=$ $(3 m / \Lambda)^{1 / 4}$; from Eq. $(162)$ it follows that for $\mathbf{a} \cdot \mathbf{v}=\mathbf{0}$ we must further put $m=1 / 3(\varepsilon / \Lambda)^{1 / 2}$ (requiring now $\varepsilon / \Lambda>0$ ), so $y=\varepsilon(\varepsilon / \Lambda)^{1 / 2}$ in the limit. This suggests inserting

$$
\begin{aligned}
m & =1 / 3(\varepsilon / \Lambda)^{1 / 2}\left(1-3 / 2 \varepsilon \delta^{2}\right), \\
y & =\varepsilon(\varepsilon / \Lambda)^{1 / 2}(1+\bar{y} \delta), \\
z & =(\varepsilon / \Lambda)^{1 / 2} \bar{z} \delta^{-1},
\end{aligned}
$$


where the parameter $\delta$ is then allowed to approach zero. The result for all three metrics is

$$
\begin{aligned}
d s^{2}= & \frac{\varepsilon}{\Lambda}\left\{-h^{2}[x+f(t)] d t^{2}+d x^{2}\right. \\
& \left.+\left(1-\varepsilon \bar{y}^{2}\right)^{-1} d \bar{y}^{2}+\left(1-\varepsilon \bar{y}^{2}\right) d \bar{z}^{2}\right\}
\end{aligned}
$$

where $h$ is the appropriate circular or hyperbolic function in each case. The $(\bar{y}, \bar{z}) 2$-spaces have constant curvature $\Lambda^{-1}$. Thus, introducing polar coordinates, we see that metric I becomes IV, as in Eq. (177), and II and III become, respectively

$$
\begin{aligned}
\text { V. } a_{\perp}= & 0, \quad \Lambda<0 \\
d s^{2}= & \frac{1}{-\Lambda}\left\{-\sinh ^{2}[x+f(t)] d t^{2}+d x^{2}+d \theta^{2}\right. \\
& \left.+\sinh ^{2} \theta d \phi^{2}\right\}
\end{aligned}
$$

$$
\begin{aligned}
& \text { VI. } a_{1}=0, \quad \Lambda<0 \\
& \qquad \begin{aligned}
d s^{2}= & \frac{1}{-\Lambda}\left\{-\cosh ^{2}[x+f(t)] d t^{2}+d x^{2}+d \theta^{2}\right. \\
& \left.+\cosh ^{2} \theta d \phi^{2}\right\}
\end{aligned}
\end{aligned}
$$

The space-time metrics I, II, and III are well known. It is easily verified that the indefinite $(x, t) 2$-spaces in all three cases have homogeneous, constant Riemannian curvatures, $\pm 1 / y^{2}$. Thus, in the case of metric I for instance, this 2-space could be written in terms of new, non-co-moving coordinates $(\theta, \bar{t})$ as

$$
y^{2}\left[-\sin ^{2} \theta d \bar{t}^{2}+d \theta^{2}\right],
$$

and metric I becomes then

$$
\begin{aligned}
d s^{2}= & y^{2}\left\{-\sin ^{2} \theta d \bar{t}^{2}+d \theta^{2}\right\} \\
& +\left(1-\frac{2 m}{y}-\frac{1}{3} \Lambda y^{2}\right)^{-1} d y^{2} \\
& +\left(1-\frac{2 m}{y}-\frac{1}{3} \Lambda y^{2}\right) d z^{2}
\end{aligned}
$$

Here the $\bar{t}$ lines are a timelike isometry of the manifold; for $\Lambda=0$, this is precisely the canonical form of a degenerate static vacuum metric of Class B-1 in the nomen- clature of Ehlers and Kundt (Ref. 15). A similar rewriting of metrics II and III, bringing them to static form, shows that II is Class B-2, while III corresponds to the class of analytically extended metrics $\mathscr{B}-2$, where the notation is again that of Ref. 15. Summarizing then we may state that, excluding spaces of constant curvature, the only Einstein spaces admitting nonisometric, irrotational, rigid congruences are the degenerate static metrics of Class B, and the special singular solutions IV, V, and VI, which require $\Lambda \neq 0$.

In the co-moving coordinates, the world lines of the rigid frame for all these metrics are the lines $t$ varies and, so long as $f$ is some function of $t$, the congruence is nonisometric. The process of direct construction we have followed demonstrates these to be the only nonisometric, irrotational, rigid congruences in Einstein spaces with nonvanishing Weyl tensor. In every case, only one arbitrary function of time remains to be specified; in other words, irrotational rigid frames in exterior gravitational fields have no more than one degree of freedom. Anticipating the results of Section VI, where it is shown that rotating rigid congruences allow no arbitrary time functions, we actually can generalize the preceding sentence by deleting the word "irrotational."

The relation between the co-moving coordinates of Eq. (174) and the static coordinates of Eq. (180) is interesting for its connection with the geometric construction for irrotational rigid motions in uniformly curved spacetime discussed in Section III. Consider a 2-space of constant, unit curvature (but of hyperbolic metric, for the present cases). We could use polar coordinates $\theta, \bar{t}$ in this 2-space so that the metric would take the nonmaximal, but simple, form

$$
d l^{2}=-\sin ^{2} \theta d \bar{t}^{2}+d \theta^{2} .
$$

If we are given a convex spacelike curve $\circlearrowright$ in this space, we may however prefer to introduce orthogonal coordinates $\bar{\theta}, t$ based on $\varrho$ by the construction on the convex side of $C$ shown in the figure. Tangent geodesics from $C$ are taken as the lines $t=$ constant. Orthogonal to them are the involutes of $\mathcal{C}$, the lines $\bar{\theta}=$ constant; the value of $\bar{\theta}$ labeling one of these is the proper distance to it along $\mathcal{C}$ from an origin $O$ on $\mathcal{C}$. If the distance along $\mathcal{C}$ from $O$ to the point of tangency of a curve $t=$ constant is $f(t)$, it follows by inspection that the form for interval $d s^{2}$ at any point $P$ is being expressed in terms of "moving polar coordinates" based on a pole that migrates along $\mathfrak{C}$ :

$$
d s^{2}=-\sin ^{2}[\bar{\theta}-f(t)] d t^{2}+d \bar{\theta}^{2}
$$


In these coordinates, the equation for $C$ itself is just $\bar{\theta}-f(t)=0$. The congruence of involutes is clearly rigid, equidistant. This construction is shown in Fig. 1.
In Eq. (174), we have this construction, if we identify $\bar{\theta}$ with $-x$. The curves $\mathcal{C}$ in the $x, t$ pseudospherical subspaces form a spacelike 3 -surface in $x, y, z, t$ space. All points in the rigid body achieve infinite acceleration.

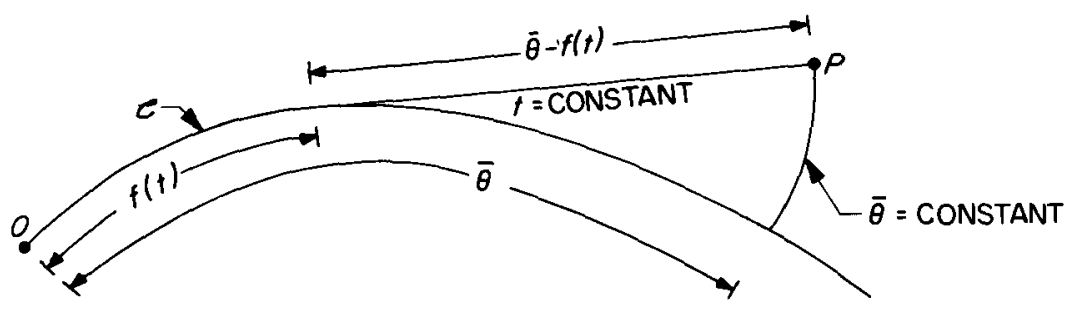

Fig. 1. Moving polar coordinates based on a curve $C$

\section{ROTATING RIGID FRAMES}

We now inquire whether any nonisometric rotating rigid motions exist and, if so, the maximum number of degrees of freedom allowed, and the form of any constraints imposed on the time dependence of the acceleration, angular velocity, or other variables.

Partial answers to some of these questions have been given in recent years (Refs. 7 to 9). Pirani and Williams (Ref. 8) show how to construct (by means of a certain metric transformation) examples of space-time metrics admitting nonisometric, rotating, rigid congruences. Thus, they have demonstrated that the Herglotz-Noether theorem is not always valid. Unfortunately, their discussion does not reveal explicitly the form of the Ricci tensor of the manifolds obtained, and it is apparently not at all clear whether the associated Einstein or stress tensor is physically reasonable. In particular, it seems not to be known whether any of their metrics include empty or Einstein spaces, nor whether the tangent vector of the rigid congruence is the timelike eigenvector of the stress tensor. As discussed in the introduction to this report, the latter are the most interesting situations physically; both cases are characterized by the vanishing of the momentum density $\mathbf{t}=0$.

In order to discuss the contributions of Rayner (Ref. 7) and Boyer (Ref. 9), we shall give up momentarily any restriction to Einstein space, and refer rather to the general dyadic equations of Appendix B, specialized only to what we have called dynamical rigid bodies $(s=0$, 
$\mathbf{t}=0$ ) with co-rotating basis triads $(\omega=\Omega)$. The dyadic $E$ is, of course, still time independent $(\dot{E}=0)$; and the Gauss equation (B-18) reads $E=-(P+3 \Omega \Omega)$, so that we have

$$
\dot{\mathbf{p}}+3 \dot{\Omega} \Omega+3 \Omega \dot{\Omega}=0 .
$$

This equation has been quoted in various forms by all the mentioned authors. Rayner combined the trace of this equation

$$
\operatorname{Tr} \dot{\mathbf{P}}+6 \Omega \cdot \dot{\Omega}=(\operatorname{Tr} \mathbf{P})^{\bullet}+3\left(\Omega^{2}\right)^{\bullet}=0
$$

with the contracted Bianchi identity equation (B-29), which for $S=t=0$ reads simply

$$
\dot{\rho}=0 \text {, }
$$

to obtain two important results: (1) the local energy density $\rho$ is time independent, and (2) since $\rho=-1 / 2 \operatorname{Tr} P$ [Eq. (B-13)], we find from Eq. (184) that

$$
\left(\Omega^{2}\right)^{\bullet}=0 \text {. }
$$

These constraints are thus valid in general for both test rigid bodies in Einstein space and dynamical rigid bodies. In the dynamical case, they would appear to be of considerable import for the problem of gravitational radiation from an asymmetric, rotating, rigid body (a spinning rod, for instance). It is difficult to see how such a system could radiate energy with these consträints.

Returning to the question of the validity of the Herglotz-Noether theorem, we can demonstrate that a sufficient condition even here is that the angular velocity vector be body-fixed, $\dot{\boldsymbol{\Omega}}=\mathbf{0}$. For it then follows directly from the antisymmetric part of $\mathrm{Eq}_{\mathrm{q}}$. $(\mathrm{B}-14)$ that $\nabla \times \mathrm{a}=0$, and from time differentiating the trace and the antisymmetric part of Eq. (B-15) that $\left(\Omega^{2}\right) \dot{\mathfrak{a}}=0$, so that for a rotating body we must have $\nabla \times \mathbf{a}=\dot{\mathbf{a}}=0$, which with $\mathbf{S}=\mathbf{0}$ are the equations characterizing a Killing vector congruence. This result can be used to obtain Boyer's (Ref. 9) sufficient, but not necessary, conditions on the curvature quantities for the validity of the HerglotzNoether theorem. In our notation, his conditions become $t=0, \mathbf{P}=1 / 3(\operatorname{Tr} \mathbf{P}) I$ and, from Eqs. (183) and (185), it is clear that these will suffice. The problem of necessary conditions, however, is still entirely open.

Returning now to the case of rotating rigid frames in Einstein space, we have obtained a complete set of differential equations of first order in the local time derivatives, which govern the evolution of these motions. Thus, we can state that, in general Einstein space, the entire history of rotating rigid motions is determined by initial conditions on any one spatial hypersurface so that no arbitrary functions of time (degrees of freedom) are permitted. In a sense, this incorporates much of the physical significance of the Herglotz-Noether theorem. Actually, as we shall see, the total set of equations is over-complete, since four subsidiary algebraic equations are obtained in addition to the differential equations. Isometric motions, for which all quantities are time independent, constitute a trivial solution of the total set; it may be that these are the only solutions consistent with the subsidiary equations, in which case the usual statement of the HerglotzNoether theorem would hold. Unfortunately, the algebraic complexity of the equations has so far prohibited either reducing them to the conditions for isometries, or, conversely, demonstrating one or more particular nonisometric solutions.

The derivation of the equations depends crucially on the commutation of the space and time derivative operators for time-independent quantities [Eq. (54)]; that these commute, as remarked in Section II-D, is a special property of rigid motions. We start again with the Gauss equation (61) for rigid motion in Einstein space

$$
E=-\mathbf{A}-3 \Omega \Omega+\frac{1}{3} \Lambda \mathbf{I}
$$

and the constraint $\dot{E}=0$. The trace of Eq. (187) gives

$$
\operatorname{Tr} \mathbf{E}=\Lambda-3 \Omega^{2},
$$

and time differentiating we find, of course, that

$$
\frac{1}{2}\left(\Omega^{2}\right)^{\bullet}=\Omega \cdot \dot{\Omega}=0 .
$$

In view of this orthogonality property, we can define for later use a vector $\sigma$, by setting

$$
\left(\Omega^{2}\right) \sigma \equiv \Omega \times \dot{\Omega}
$$

and in consequence express $\dot{\Omega}$ as

$$
\dot{\boldsymbol{\Omega}}=\boldsymbol{\sigma} \times \boldsymbol{\Omega},
$$

where by definition

$$
\boldsymbol{\sigma} \cdot \Omega=0 .
$$


Differentiating this last equation, we can show that

$$
\dot{\boldsymbol{\sigma}} \cdot \Omega=0 .
$$

The procedure now is to construct additional timeconstant dyadics from the spatial derivatives of $\mathbf{E}$. The existence of a chain of such quantities resulting from repeated spatial differentiation was previously pointed out by Pirani and Williams (Ref. 8), though not exploited by them. The success of this procedure depends on the fact that the differentiation process may be selectively applied to obtain only equations which do not contain spatial derivatives of time-dependent quantities; furthermore, with this criterion, the procedure terminates naturally, so that finally a complete set results, all first-order differential equations in time only.

We first define the dyadic $\mathbf{F}$ by

$$
F \equiv-\nabla \times E=F^{\prime \prime}-\mathbf{f} \times \mathbf{I},
$$

where $F^{*}$ and $\mathbf{f}$ are respectively symmetric and antisymmetric parts of $\mathbf{F}$, and by commutation $(\dot{\mathbf{E}}=0)$ it follows that

$$
\dot{\mathbf{f}}=\dot{\boldsymbol{F}}^{*}=\dot{\mathbf{f}}=0 .
$$

Some of the other properties of $\mathbf{F}$ can be obtained before calculating its explicit form. Since $E$ is symmetric, we have $\operatorname{Tr} F=\operatorname{Tr} \boldsymbol{F}^{*}=0$ by Eq. (A-28); by using (A-27), (188), and the fact that $\nabla \cdot E=0$, we find

$$
\mathbf{f}=\frac{1}{2} \nabla(\operatorname{Tr} \mathbf{E})=-\frac{3}{2} \nabla\left(\Omega^{2}\right) .
$$

From this and the commutation relation in Eq. (55), it follows that

$$
\nabla \times f=0 .
$$

Now taking the divergence of Eq. (194) gives

$$
\nabla \cdot F=-\nabla \cdot(\nabla \times E)=\nabla \cdot F^{s}-\nabla \times f,
$$

but from the commutation relation equation (D-57) of Ref. 10, we have

$$
\nabla \cdot \nabla \times E=-E_{x} \cdot E+2 \Omega \cdot \dot{E}=0
$$

so that finally also

$$
\boldsymbol{\nabla} \cdot \boldsymbol{F}^{s}=\mathbf{0}
$$

We obtain the explicit expression for $\mathbf{F}$ by substituting from Eqs. (66), (68), and (70) into

$$
\begin{aligned}
\mathbf{F} & =-\nabla \times \mathbf{E}=\nabla \times\left(\mathbf{A}+3 \Omega \Omega-\frac{1}{3} \Lambda \mathbf{l}\right) \\
& =\nabla \times \mathbf{A}+3 \nabla \times \Omega \Omega-3 \Omega \times \nabla \Omega,
\end{aligned}
$$

and upon resolving find for $F^{s}$ and $f$

$$
\begin{aligned}
\mathbf{F}^{s}= & \dot{\mathbf{B}}-\boldsymbol{\Omega} \times \mathbf{B}+\mathbf{B} \times \Omega+\mathbf{a} \times \mathbf{E}-\mathbf{E} \times \mathbf{a} \\
& +3 \Omega \Omega \times \mathbf{a}-3 \mathbf{a} \times \Omega \Omega, \\
& \frac{1}{3} \mathbf{f}=2\left(\Omega^{2}\right) \mathbf{a}-(\mathbf{a} \cdot \Omega) \Omega-\Omega \cdot \mathbf{B} .
\end{aligned}
$$

Proceeding to the next level of spatial differentiation, we define the dyadic $\mathbf{G}$ by

$$
\mathbf{G} \equiv \nabla \times\left[F^{*}+\frac{1}{3} \mathbf{f} \times \mathbf{I}\right]=\mathbf{G}^{s}-\mathbf{g} \times \mathbf{I}
$$

and as before, of course, have

$$
\dot{\mathbf{G}}=\dot{\mathbf{G}} s=\dot{\mathbf{g}}=0 .
$$

Using Eq. (A-27), we find that

$$
\mathbf{g}=\frac{1}{2}\left[\boldsymbol{\nabla} \cdot \mathbf{F}^{\times}-\frac{1}{3} \nabla \times \mathbf{f}-\boldsymbol{\nabla}\left(\operatorname{Tr} \mathbf{F}^{*}\right)\right]=0,
$$

so that $\mathbf{G}$ is symmetric; and tracing Eq. (201) we have according to Eq. (A-28)

$$
\operatorname{Tr} \mathbf{G}=\operatorname{Tr} \mathbf{G}^{s}=-\frac{2}{3} \boldsymbol{\nabla} \cdot \mathbf{f} .
$$

To calculate the explicit form of G", Eqs. (199) and (200) are inserted in Eq. (201), and then, using virtually all the dyadic equations of Section II-D, it is possible to eliminate the spatial derivatives of all time-dependent quantities. This was the criterion for the particular choice of G. To assist in the elimination, it is convenient to introduce a new, symmetric, time constant, dyadic $\mathbf{G}^{K}$ by

$$
\begin{aligned}
\mathbf{G}^{\kappa} \equiv \mathbf{G}^{S}+3 \mathbf{E} \cdot \mathbf{E}-3\left(\Lambda-\Omega^{2}\right) \mathbf{E} \\
-\left[\mathbf{E}: \mathbf{E}-10 \Omega^{4}+\frac{23}{3} \Lambda \Omega^{2}-\Lambda^{2}\right] 1 .
\end{aligned}
$$


The lengthy result of all the substitutions then becomes

$$
\begin{aligned}
& \mathbf{G}^{K}=3 \dot{\sigma} \times \Omega \Omega-3 \Omega \Omega \times \dot{\boldsymbol{\sigma}}-\dot{\mathbf{a}} \times \mathbf{B}+\mathbf{B} \times \dot{\mathbf{a}} \\
& +(\boldsymbol{\sigma} \times \Omega) \times \mathbf{E}-\mathbf{E} \times(\boldsymbol{\sigma} \times \Omega) \\
& +6 \sigma \times \Omega \sigma \times \Omega-6 \sigma^{2} \Omega \Omega \\
& -2 \Omega^{2}(\sigma \Omega+\Omega \sigma)-5 \Omega^{2} \mathbf{a a} \\
& +\left(2 \Omega^{2}+\frac{4}{3} a^{2}-\frac{8}{3} \Lambda\right) \Omega \Omega \\
& +\left[\mathbf{E}+\frac{4}{3} \Omega \Omega-\frac{1}{3} \Lambda \mathbf{I}\right] \times\left[3 \mathbf{a a}+2 \Omega \Omega+a^{2} \mathbf{I}\right] \\
& -2[B+(a \cdot \Omega) I-2 a \Omega]_{x}^{\times}[B+(a \cdot \Omega) I-2 a \Omega]-a \cdot \mathcal{E} \\
& -2 \mathbf{a} \times\left[\mathbf{F}^{s}+\frac{1}{3} \mathbf{f} \times \mathbf{I}\right]+2\left[\mathbf{F}^{s}-\frac{1}{3} \mathbf{f} \times \mathbf{I}\right] \times \mathbf{a} \\
& +\frac{1}{3}\left[20 a^{2} \Omega^{2}-\Lambda a^{2}-9 \Omega \cdot E \cdot \Omega+3 a \cdot E \cdot a\right] \text {, }
\end{aligned}
$$

where the symbol $\delta$ denotes the time-constant triadic $\mathcal{S}=$ PE. Taking the trace of this equation, we have

$$
\operatorname{Tr} \mathbf{G}^{K}=2 \mathrm{~B}: \mathbf{B}-8 \Omega \cdot \mathbf{E} \cdot \mathbf{\Omega}+\frac{10}{3} \mathbf{a} \cdot \mathbf{f}+5 \Omega^{2}\left(\Omega^{2}-\Lambda\right) .
$$

This completes the derivation of the equations. They are all expressed in terms of the following two sets of quantities: (1) a, $\Omega, \boldsymbol{\sigma}, \mathbf{B}$; and (2) $\mathbf{E}, \mathbf{F}^{S}, \mathbf{f}, \mathbf{G}^{K}, \mathcal{E}$. The second set contains only quantities whose time derivatives vanish; they can, therefore, be treated as arbitrary constants whose values may be selected at any point on each world line of the congruence. The first set contains 14 scalars whose time dependence is governed, either explicitly or implicitly, by the 14 scalar equations included in Eqs. (191), (193), (199), and the trace-free part of Eq. (206). These equations are linear in, and could in principle all be explicitly solved for, all the first time derivatives. This is indeed already accomplished for $\dot{\boldsymbol{\Omega}}$ and $\dot{\mathbf{B}}$. Further differentiation and substituting back would then generate the time derivatives of all order in terms of undifferentiated quantities, so that the entire evolution on each line is determined by conditions at one point.

We have four algebraic constraints left over: Eqs. (200) and (207). These must hold throughout time so that, by differentiating them and substituting back for all time derivatives, we should obtain a further set of four (in general new) algebraic constraints. These, in turn, could be treated in identical fashion, and so on and on. If this process continues to generate a chain of independent equations, we should soon be able to solve algebraically for $\mathbf{a}, \Omega, \sigma$, and $\mathbf{B}$ in terms of the second, time-constant, set of quantities, and so all the quantities would be time constant, and we would have accomplished the reduction to isometric motions. This, however, remains to be done, and so we cannot exclude the possibility that a very few, very special, solutions might exist, for which the above described chain of algebraic manipulation would stop, self-consistent, but short of the complete reduction to a timelike isometry. 


\section{APPENDIX A \\ Summary of Dyadic Notation and Identities}

For reference purposes, we summarize briefly the dyadic notation and list some identities used in the paper. Let

$$
\mathbf{u}^{a}=\mathbf{u}_{a}, \quad a=1,2,3
$$

be an orthonormal triad of basis vectors. The index $a$ simply labels the vectors and is freely written up or down as convenient. Using the dot notation for the scalar product and the Kronecker delta, the orthonormality relations read

$$
\mathbf{u}^{a} \cdot \mathbf{u}^{b}=\delta^{a b} .
$$

Introducing the usual vector cross-product notation and the numerical permutation symbol, we write

$$
\mathbf{u}_{a} \times \mathbf{u}_{b}=\varepsilon_{a b c} \mathbf{u}^{c}, \quad \mathbf{u}^{a}=\frac{1}{2} \varepsilon^{a b c} \mathbf{u}_{b} \times \mathbf{u}_{\mathrm{c}},
$$

where the summation convention for repeated indices always applies unless specifically revoked. The unit dyadic may then be written as

$$
\mathbf{I}=\mathbf{u}^{a} \mathbf{u}^{a}
$$

In the following, the symbols $\mathbf{V}$ and $\mathbf{W}$ stand for arbitrary vectors, and $\boldsymbol{M}, \mathbf{N}$, and $\mathbf{P}$ for arbitrary dyadics. Expanding in terms of the basis vectors,

$$
\begin{aligned}
& \mathbf{V}=\mathbf{V} \cdot \mathbf{I}=\left(\mathbf{V} \cdot \mathbf{u}_{a}\right) \mathbf{u}^{a}=V_{a} \mathbf{u}^{a}, \\
& \mathbf{M}=\mathbf{I} \cdot \mathbf{M} \cdot \mathbf{I}=\mathbf{u}^{a} \mathbf{u}_{a} \cdot \mathbf{M} \cdot \mathbf{u}_{b} \mathbf{u}^{b}=\boldsymbol{M}_{\mathbf{a} b} \mathbf{u}^{a} \mathbf{u}^{b},
\end{aligned}
$$

defines the orthonormal components, $V_{a}$ and $M_{a b}$. $\operatorname{Tr} M$ denotes the contraction $M_{a}^{a}$, and the superscript $T$ denotes a transposed dyadic; $M^{T}=M_{b a} \mathbf{u}^{a} \mathbf{u}^{b}$. Using the expansions and Eqs. (A-1) and (A-2), any dyadic expression can be quickly converted to component form and identities derived. We have, for instance,

$$
\begin{aligned}
& \mathbf{V} \times \mathbf{M}=V_{c} M_{d b} \mathbf{u}^{c} \times \mathbf{u}^{d} \mathbf{u}_{b}=\left(\varepsilon^{a c d} V_{c} M_{d b}\right) \mathbf{u}_{a} \mathbf{u}_{b}, \\
& \mathbf{M} \times \mathbf{V}=\left(\varepsilon^{b c d} M_{a c} V_{d}\right) \mathbf{u}_{a} \mathbf{u}_{b},
\end{aligned}
$$

with the special case

$$
\mathbf{V} \times \mathbf{I}=\mathbf{I} \times \mathbf{V}=\left(\varepsilon^{a c b} V_{c}\right) \mathbf{u}_{a} \mathbf{u}_{b} .
$$

The transpose identity for these is

$$
(\mathbf{V} \times \mathbf{M})^{T}=-\mathbf{M}^{T} \times \mathbf{V},
$$

and

$$
(\mathbf{V} \times I)^{T}=-1 \times V=-V \times I .
$$

Several double operation symbols are used, with the convention that the upper operation acts first:

$$
\begin{aligned}
& \mathbf{M}: \mathbf{N}=\operatorname{Tr}(\mathbf{M} \cdot \mathbf{N})=M^{a b} N_{b a}, \\
& \mathbf{M}_{\mathbf{x}} \mathbf{N}=\left(\boldsymbol{\varepsilon}^{a b c} \mathbf{M}_{b d} N_{d c}\right) \mathbf{u}_{a}=-\mathbf{N}^{T} \cdot \mathbf{M}^{T} .
\end{aligned}
$$

In addition, the dyadic symbol $\boldsymbol{M}_{x} \mathbf{N}$ is very convenient, but requires a special convention; using the upper cross first to operate on the "inside" vectors, we let the resulting vector stand to the left. Thus,

$$
\begin{aligned}
\mathbf{M}_{\mathbf{\times}}^{\mathbf{X}} \mathbf{N} & =M_{c d} N_{f g} \mathbf{u}^{c} \mathbf{u}_{\times}^{d \mathbf{x}} \mathbf{u}^{\prime} \mathbf{u}^{g}=M_{c d} N_{f g} \mathbf{u}^{d} \times \mathbf{u}^{f} \mathbf{u}^{c} \times \mathbf{u}^{g} \\
& =\left(\varepsilon_{a d f} \varepsilon_{b c g} M_{c d} N_{f g}\right) \mathbf{u}_{a} \mathbf{u}_{b}
\end{aligned}
$$

and the following relations hold

$$
\mathbf{M}_{\times}^{\times} \mathbf{N}=\mathbf{N}_{\times}^{T \times} \mathbf{M}^{T}, \quad\left(\mathbf{M}_{\times}^{\times} \mathbf{N}\right)^{T}=\mathbf{N}_{\times}^{\times} \mathbf{M} .
$$

The double permutation symbol may be expanded to give an identity which is best written by transposing the second dyadic:

$$
\begin{aligned}
\mathbf{M}_{\mathbf{X}}^{\mathbf{X}} \mathbf{N}^{r}= & \mathbf{M} \cdot \mathbf{N}+\mathbf{N} \cdot \mathbf{M}-(\operatorname{Tr} \mathbf{M}) \mathbf{N}-(\operatorname{Tr} \mathbf{N}) \mathbf{M} \\
& +[(\operatorname{Tr} \mathbf{M})(\operatorname{Tr} \mathbf{N})-\mathbf{M}: \mathbf{N}] \mathbf{I},
\end{aligned}
$$

and so for the trace

$$
\operatorname{Tr}\left(\mathbf{M}_{\mathbf{X}}^{\mathbf{X}} \mathbf{N}^{r}\right)=(\operatorname{Tr} \mathbf{M})(\operatorname{Tr} \mathbf{N})-\mathbf{M}: \mathbf{N} .
$$

Resolving a dyadic $M$ into symmetric and antisymmetric parts, we may always write

$$
\mathbf{M}=\mathbf{M}^{s}-\mathbf{m} \times \mathbf{l}, \quad \mathbf{M}^{r}=\mathbf{M}^{s}+\mathbf{m} \times \mathbf{I},
$$

where $\mathbf{M}^{S} \equiv 1^{1 / 2}\left(\boldsymbol{M}+\mathbf{M}^{T}\right)$, and we have introduced the dual vector $m$ defined by

$$
\mathbf{m} \equiv \frac{1}{2} M_{x} 1=\frac{1}{2}\left(\varepsilon^{a b c} M_{b c}\right) \mathbf{u}_{a}
$$


to represent the antisymmetric part,

$$
\frac{1}{2}\left(\mathbf{M}-\mathbf{M}^{T}\right)=-\mathbf{m} \times \mathbf{l} \text {. }
$$

Two dyadic identities often used are analogous to the corresponding vector formulas for transforming triple products:

$$
\begin{aligned}
\mathbf{W} \cdot(\mathbf{V} \times \mathbf{M}) & =(\mathbf{W} \times \mathbf{V}) \cdot \mathbf{M}, \\
\mathbf{W} \times(\mathbf{V} \times \mathbf{M}) & =\mathbf{V} \mathbf{W} \cdot \mathbf{M}-(\mathbf{W} \cdot \mathbf{V}) \mathbf{M},
\end{aligned}
$$

with the special cases

$$
\begin{aligned}
\mathbf{W} \cdot(\mathbf{V} \times \mathbf{I}) & =\mathbf{W} \cdot(\mathbf{I} \times \mathbf{V})=\mathbf{W} \times \mathbf{V} \\
\mathbf{W} \times(\mathbf{V} \times \mathbf{I}) & =\mathbf{W} \times \mathbf{I} \times \mathbf{V}=\mathbf{V} \mathbf{W}-(\mathbf{V} \cdot \mathbf{W}) \mathbf{I}
\end{aligned}
$$

Also,

$$
\mathbf{V} \times \mathbf{M} \times \mathbf{W}=-(\mathbf{W V})_{\times}^{\mathbf{X}} \mathbf{M}=-\mathbf{M}_{\mathbf{x}}^{\mathrm{TX}}(\mathbf{V W}),
$$

where the general expansion of the double cross product can then be applied to the latter forms.

Some useful composite identities are:

$$
\begin{aligned}
& (\mathbf{M} \cdot \mathbf{N})_{\dot{x}}^{\dot{P}}=\mathbf{M}_{\mathbf{x}}^{\dot{*}}(\mathbf{N} \cdot \mathbf{P}), \\
& (\mathbf{V} \times \mathbf{M}): \mathbf{N}=\mathbf{V} \cdot\left(\mathbf{M}_{\mathbf{x}} \mathbf{N}\right), \\
& (\mathbf{V} \times \mathbf{M})_{\dot{x}} \mathbf{N}=\mathbf{N}_{\mathbf{x}}^{T}\left(\mathbf{M}^{T} \times \mathbf{V}\right)=\mathbf{M} \cdot \mathbf{N} \cdot \mathbf{V}-(\mathbf{M}: \mathbf{N}) \mathbf{V}, \\
& \mathbf{N}_{\mathbf{x}}(\mathbf{V} \times \mathbf{M})=\left(\mathbf{M}^{T} \times \mathbf{V}\right)_{\mathbf{x}}^{\dot{N^{T}}}=-\mathbf{V} \cdot\left(\mathbf{N}_{\mathbf{x}}^{\mathbf{X}} \mathbf{M}\right), \\
& \left(\mathbf{M}_{\mathbf{X}}^{\times} \mathbf{N}^{T}\right): \mathbf{P}=\left(\mathbf{P}_{\mathbf{X}}^{\times} \mathbf{M}^{T}\right): \mathbf{N}=\left(\mathbf{N}_{\mathbf{x}}^{\times} \mathbf{P}^{T}\right): \mathbf{M}, \\
& \left(\mathbf{M}_{\mathbf{x}}^{\mathbf{x}} \mathbf{N}^{T}\right)_{\dot{x}} \mathbf{P}=-\left(\mathbf{M}_{\mathbf{x}} \mathbf{P}^{T}\right) \cdot \mathbf{N}-\left(\mathbf{N}_{\mathbf{x}} \mathbf{P}^{T}\right) \cdot \mathbf{M} .
\end{aligned}
$$

Note also that we can write

$$
\operatorname{Tr}(\mathbf{V} \times \mathbf{M})=(\mathbf{V} \times \mathbf{M}): \mathbf{I}=\mathbf{V} \cdot\left(\mathbf{M}_{\mathbf{x}}^{\mathbf{l}} \mathbf{l}\right)=2 \mathbf{V} \cdot \mathbf{m},
$$

which therefore vanishes if $M$ is symmetric.
The algebra of the $\boldsymbol{\nabla}$ operator generates many familiar vector and dyadic identities which, in the dyadic case, grow from the seed formulas

$$
\boldsymbol{\nabla} \cdot(\psi \mathbf{V W})=(\boldsymbol{\nabla} \psi) \cdot \mathbf{V W}+\psi(\boldsymbol{\nabla} \cdot \mathbf{V}) \mathbf{W}+\psi \mathbf{V} \cdot \boldsymbol{\nabla} \mathbf{W},
$$

$\boldsymbol{\nabla} \times(\psi \mathbf{V W})=(\boldsymbol{\nabla} \psi) \times \mathbf{V W}+\psi(\nabla \times \mathbf{V}) \mathbf{W}-\psi \mathbf{V} \times \boldsymbol{\nabla} \mathbf{W}$

where $\psi$ is an arbitrary scalar. Not-so-familiar ones found useful in the derivations in this report are

$$
\begin{aligned}
& \boldsymbol{\nabla} \cdot\left(\mathbf{M}_{\mathbf{x}} \mathbf{N}\right)=(\boldsymbol{\nabla} \times \mathbf{M}): \mathbf{N}+\mathbf{M}:(\mathbf{N} \times \boldsymbol{\nabla}), \\
& \boldsymbol{\nabla} \cdot\left(\boldsymbol{M}_{\mathbf{x}}^{\mathbf{x}} \mathbf{N}^{T}\right)=-\mathbf{N}_{\mathbf{x}}^{\dot{*}}\left(\boldsymbol{\nabla} \times \mathbf{M}^{T}\right)-\mathbf{M}_{\mathbf{x}}\left(\boldsymbol{\nabla} \times \mathbf{N}^{T}\right), \\
& \boldsymbol{\nabla} \cdot(\mathbf{V} \times \mathbf{M})=-\mathbf{V} \cdot(\boldsymbol{\nabla} \times \mathbf{M})+(\boldsymbol{\nabla} \times \mathbf{V}) \cdot \mathbf{M} \\
& \boldsymbol{\nabla} \cdot(\mathbf{M} \times \mathbf{V})=(\nabla \cdot \mathbf{M}) \times \mathbf{V}+\mathbf{M}_{\mathbf{x}}^{T}(\nabla \mathbf{V}), \\
& \boldsymbol{\nabla} \times(\mathbf{V} \times \mathbf{M})=(\mathbf{V} \boldsymbol{\nabla}) \cdot \mathbf{M}-(\boldsymbol{\nabla} \cdot \mathbf{V}) \mathbf{M} \\
& +\mathbf{V}(\boldsymbol{\nabla} \cdot \mathbf{M})-\mathbf{V} \cdot \boldsymbol{\nabla} \mathbf{M} \\
& \boldsymbol{\nabla} \times(\mathbf{M} \times \mathbf{V})=(\boldsymbol{\nabla} \times \mathbf{M}) \times \mathbf{V}-\mathbf{M}_{\mathbf{x}}^{T \mathbf{X}}(\boldsymbol{\nabla} \mathbf{V}) .
\end{aligned}
$$

Identities involving second-order derivatives become complicated and unfamiliar, since they must take account of the noncommutative properties of the $\boldsymbol{\nabla}$ operator in curved spaces with anholonomic basis vectors. In practice, only the commutation relations themselves (Appendix B) are ever really needed.

Finally, some special applications of the dual vector representation of antisymmetric parts arc

$$
\begin{aligned}
\nabla \mathbf{V}= & \frac{1}{2}(\nabla \mathbf{V}+\mathbf{V} \nabla)-\frac{1}{2}(\nabla \times V) \times I, \\
\nabla \times M= & \frac{1}{2}\left[\nabla \times M-M^{T} \times \nabla\right] \\
& -\frac{1}{2}\left[\nabla \cdot M^{T}-\nabla(\operatorname{Tr} M)\right] \times 1,
\end{aligned}
$$

and

$$
\operatorname{Tr}(\nabla \times M)=\nabla \cdot\left(M_{x}^{\prime} l\right)=2 \nabla \cdot m,
$$

so that if $\mathbf{M}$ is symmetric, $\operatorname{Tr}(\nabla \times M)=0$. 


\section{APPENDIX B Summary of the General Dyadic Equations}

The general dyadic equations for a timelike congruence were derived in Ref. 10 by introducing an orthonormal tetrad field aligned so that the timelike tetrad vector everywhere coincides with the unit tangent to the congruence. The tetrad vectors were denoted by their components, contravariant ${ }_{2} \lambda^{\mu}$ and covariant ${ }^{r} \lambda_{\mu}$, in an arbitrary holonomic coordinate system $x^{\mu}(\mu=1,2,3,4)$, where the Latin index $(r=0,1,2,3)$ labels the vectors. In this notation, the orthonormality relations become

$$
{ }_{r} \lambda^{\mu s} \lambda_{\mu}=\delta_{r}^{s}, \quad{ }_{,} \lambda^{\mu r} \lambda_{\nu}=\delta_{\nu}^{\mu}
$$

${ }_{(1,} \lambda^{\mu}$ is the unit tangent vector to the timelike congruence and ${ }_{n} \lambda^{\mu}(a=1,2,3)$ are the spacelike triad vectors. These quantities may alternatively be viewed as the transformation matrices between two sets of vector bases; one set comprises the natural holonomic basis vectors associated with a coordinate system $x^{\mu}$, the other is composed of orthonormal tetrads which will, in general, be anholonomic. Using them in this latter sense, any tensor, say $F_{\mu \nu}$, written in the usual coordinate components is converted to orthonormal components, $F_{r s}$, by

$$
F_{r s}=F_{\mu \nu r} \lambda^{\mu}{ }_{s} \lambda^{\mu}
$$

and the inverse transformation follows directly from the orthonormality relations;

$$
F_{\mu v}=F_{r *}{ }^{r} \lambda_{\mu}{ }^{*} \lambda_{v}
$$

When the coordinates $x^{\mu}$ are co-moving, the matrix of contravariant tetrad vector components takes an especially simple and convenient form; in Section II-D of this report, special notation is then introduced for the timelike components:

$$
{ }_{\lambda} \lambda^{\mu}=\left(\begin{array}{c:c}
{ }_{\lambda} \lambda^{\alpha} & { }_{a} A \\
\hdashline 0 & \phi
\end{array}\right)
$$

The indices $\alpha, a$ are from the first portions of their respective alphabets and so are understood to take the restricted range $1,2,3$. Also the reciprocal matrix is then

$$
{ }^{r} \lambda_{\mu}=\left(\begin{array}{c:c}
{ }^{a} \lambda_{\alpha} & 0 \\
\hdashline-\phi^{-1} A_{\alpha} & \phi^{-1}
\end{array}\right),
$$

where

$$
{ }_{a} A={ }_{a} \lambda^{\alpha} A_{\alpha} .
$$

The ${ }_{a} A$ and $A_{\alpha}$ are orthonormal and natural components, respectively, of a 3-vector $A$ which, with the scalar $\phi$, is fully discussed in dyadic terms in Section II-D.

The differential characteristics of the timelike congruence are expressed in the absolute acceleration and angular velocity vectors and the symmetric rate-of-strain tensor, defined as usual by

$$
\left.\begin{array}{rl}
a_{\mu} & \equiv{ }_{0} \lambda_{\mu ; \nu 0} \lambda^{v}, \\
\Omega^{\mu} & \equiv \frac{1}{2}(-g)^{-1 / 3} \varepsilon^{\mu \nu \sigma \tau}{ }_{0} \lambda_{\nu ; \sigma} \lambda_{\tau}, \\
\sigma_{\mu \nu} & \equiv{ }_{0} \lambda_{(\mu ; v)}+a_{\left(\mu_{0} \lambda_{\nu)} .\right.} .
\end{array}\right\}
$$

All these quantities are projected into the three-space orthogonal to the timelike congruence, i.e.,

$$
a^{\mu}{ }_{1} \lambda_{\mu}=\Omega^{\mu}{ }_{0} \lambda_{\mu}=\sigma^{\mu \nu}{ }_{0} \lambda_{\mu}=0 .
$$

After transforming to orthonormal components $\left(a_{a}, \Omega_{a}, \sigma_{a b}\right)$ as in Eq. (B-2), the corresponding three-dimensional vector and dyadic quantities are defined by

$$
\begin{aligned}
& \mathbf{a}=a_{a} \mathbf{u}^{a}, \quad \mathbf{\Omega}=\Omega_{a} \mathbf{u}^{a} \\
& \mathbf{S}=\mathrm{S}_{a b} \mathbf{u}^{a} \mathbf{u}^{b}=\sigma_{a b} \mathbf{u}^{a} \mathbf{u}^{b}=\mathbf{S}^{T},
\end{aligned}
$$

where $\mathbf{u}^{a}$ is the orthonormal triad of spacelike vectors with coordinate components ${ }_{a} \lambda^{\mu}$. In like manner, the various derivatives of these triad vectors are represented by a 3-vector $\omega$,

$$
\boldsymbol{\omega}=\omega_{a} \mathbf{u}^{a}, \quad \omega_{a}=\frac{1}{2} \varepsilon_{a b c} \lambda^{\mu}{ }_{b} \lambda_{\mu ; \nu 0} \lambda^{\nu},
$$

and a dyadic $\mathbf{N}$,

$$
\mathbf{N}=N_{a b} \mathbf{u}^{a} \mathbf{u}^{b}, \quad N_{a b}=\frac{1}{2} \varepsilon_{b c d c} \lambda^{\mu}{ }_{d} \lambda_{\mu ; \boldsymbol{v} a} \lambda^{\nu} .
$$

The vector $\omega$ is the angular velocity of the orthonormal triad vectors relative to Fermi propagated axes, and the dyadic $\mathbf{N}$ is the three-dimensional anholonomic affinity. (See Section II.) 
When these dyadic quantities are differentiated, both along and orthogonal to the timelike congruence, and second derivatives of the tetrad vectors are eliminated by means of the commutation law for covariant differentiation, components of the Riemann curvature tensor, $R_{. v \sigma \tau}^{\mu}$, are introduced. Two equivalent dyadic sets are employed to represent the 20 independent components of this tensor. Both are obtained as usual by taking orthonormal components. The first is straightforward and results in three symmetric dyadics $\mathbf{P}, \mathbf{Q}, \mathbf{B}$ (with $\operatorname{Tr} \mathbf{B}=0$ ) and a vector $t$ where

$$
\left.\begin{array}{rl}
P_{a b} & =-\frac{1}{4} \varepsilon_{a c d} \varepsilon_{b f g} R^{c d f g}, \\
Q_{a b} & =R_{0 a 0 b}, \\
B_{a b}-\varepsilon_{a c b} t^{c} & =\frac{1}{2} \varepsilon_{b c d} R_{0 a c d} .
\end{array}\right\}
$$

The second representation depends on the invariant resolution of the curvature tensor into the Einstein tensor $R_{\mu \nu}-1 / 2 R g_{\mu \nu}$ and Weyl's conformal tensor, $C_{. v \sigma \tau}^{\mu}$. The dyadic $B$ and the vector $t$ occur again and are thus common to both sets. Here we obtain, in addition to these, two symmetric dyadics $T, A$ (with $\operatorname{Tr} \mathbf{A}=0$ ) and a scalar $\rho$ :

$$
\left.\begin{array}{rl}
A_{a b} & =C_{0 a 0 b}=-\frac{1}{4} \varepsilon_{a c d} \varepsilon_{b f g} C^{c d f g}, \\
B_{a b} & =\frac{1}{2} \varepsilon_{b c d} C_{0 a c d}, \\
T_{a b} & =\frac{1}{2}\left(R_{a b}-\frac{1}{2} R \delta_{a b}\right), \\
t_{a} & =\frac{1}{2} R_{0 a}, \\
\rho & =-\frac{1}{2}\left(R_{00}+\frac{1}{2} R\right) .
\end{array}\right\}
$$

A and B have been called, respectively, the "electric" and "magnetic" components of the Weyl tensor. Invoking Einstein's field equations we identify $T$ as the material stress dyadic, $t$ the momentum density vector, and $\rho$ the energy density. The relations between the two sets are

$$
\left.\begin{array}{l}
\mathbf{A}=\frac{1}{2}\left[\mathbf{P}+\mathbf{Q}-\frac{1}{3}(\operatorname{Tr} \mathbf{P}+\operatorname{Tr} \mathbf{Q}) \mathbf{I}\right], \\
\mathbf{T}=\frac{1}{2}[-\mathbf{P}+\mathbf{Q}-(\operatorname{Tr} \mathbf{Q}) \mathbf{I}], \\
\boldsymbol{\rho}=-\frac{1}{2} \operatorname{Tr} \mathbf{P} .
\end{array}\right\}
$$

We use the three-dimensional $\nabla$ symbol for projected covariant differentiation orthogonal to the timelike congruence, and a superior dot for the proper time derivative along the timelike congruence. These operations are carefully defined in Ref. 10. For vectors and dyadics, this dot derivative is not covariant; it signifies the derivative of the components in an orthonormal basis triad. The vector $\dot{\mathbf{V}}+\boldsymbol{\omega} \times \mathbf{V}$ and the dyadic $\dot{\mathbf{M}}+\boldsymbol{\omega} \times \mathbf{M}-\mathbf{M} \times \boldsymbol{\omega}$ are covariant for any transformation of the basis triads (leaving the timelike direction unchanged); they are, in fact, the derivatives with respect to basis triads which are Fermi propagated $(\omega=0)$ along the timelike congruence.

With these definitions, we may write down the 36 general dyadic equations for timelike congruences:

$$
\begin{aligned}
& \boldsymbol{\nabla} \mathbf{a}-(\dot{\mathbf{s}}+\boldsymbol{\omega} \times \mathbf{s}-\mathbf{s} \times \boldsymbol{\omega})+(\dot{\boldsymbol{\Omega}}+\boldsymbol{\omega} \times \boldsymbol{\Omega}) \times \mathbf{I}= \\
& \mathbf{s} \cdot \mathbf{s}-\mathbf{\Omega} \times \mathbf{s}-\mathbf{s} \times \mathbf{\Omega}+\mathbf{\Omega}-\left(\Omega^{2}\right) \mathbf{I}-\mathbf{a} \mathbf{a}+\mathbf{Q}, \\
& \boldsymbol{\nabla} \boldsymbol{\Omega}+\mathbf{s} \times \boldsymbol{\nabla}=-\mathbf{2} \mathbf{\Omega}+(\mathbf{a} \cdot \boldsymbol{\Omega}) \mathbf{I}+\mathbf{B}+\mathbf{t} \times \mathbf{I},(\mathrm{B}-15) \\
& \dot{\mathbf{N}}+\mathbf{S}^{\star} \cdot \mathbf{N}=\mathbf{S}^{\star T} \times(\boldsymbol{\nabla}+\mathbf{a})+[(\boldsymbol{\nabla}+\mathbf{a}) \cdot(\boldsymbol{\Omega}-\boldsymbol{\omega})] \mathbf{I},
\end{aligned}
$$

$$
\nabla \times N=-\frac{1}{2} N^{2} \times \mathbf{N}+E+\left(s 2 \cdot 5^{*}\right) \times 1 .
$$

where $s^{*}=s \quad\left(\begin{array}{ll}\Omega & w\end{array}\right) \times !$. Further relations can be obtained by tracing these equations and resolving into symmetric and antisymmetric parts. In the last equation, we have introduced the symmetric dyadic $\mathbf{E}$ defined by

$$
E \equiv-P-\frac{1}{2} s_{\times}^{\times} s-\Omega \Omega-\omega \Omega-\Omega \omega .
$$

This is actually a generalized Gauss equation.

If the timelike congruence is irrotational $(\Omega=0)$, it is normal to a family of three-dimensional immersed subspaces whose second fundamental form is just $\mathbf{S}$. The dyadic $\mathbf{E}$ is then precisely the conservative, or Einstein, tensor of the 3-spaces, satisfying the Bianchi identity $\boldsymbol{\nabla} \cdot \mathbf{E}=\mathbf{0}$. Since in three dimensions the curvature tensor has only six independent components, it is completely expressed in terms of $\mathbf{E}$ by

$$
{ }^{(3)} R_{a b c d}=\varepsilon_{a b f} \varepsilon_{c d g} E^{f g} .
$$

The general commutation relations for space and time differentiation are rather complicated in this formalism 
because of the anholonomic nature of the reference systems. The following list is representative:

$$
\begin{aligned}
& (\boldsymbol{\nabla} \psi)^{\bullet}-\boldsymbol{\nabla}(\dot{\psi})=\mathbf{a} \dot{\psi}-\mathbf{s}^{\star} \cdot \boldsymbol{\nabla} \psi, \\
& (\boldsymbol{\nabla V})^{\bullet}-\boldsymbol{\nabla}(\dot{\mathbf{V}})=\mathbf{a} \dot{\mathbf{V}}-\mathbf{s}^{\star} \cdot \boldsymbol{\nabla V} \\
& -\left(\dot{\mathbf{N}}+\mathbf{S}^{\star} \cdot \mathbf{N}\right) \times \mathbf{V}, \\
& (\nabla \times M)^{\bullet}-\nabla \times(\dot{M})=a \times \dot{M}-S^{\star} \dot{x} \nabla M \\
& -\left(\dot{\mathbf{N}}+\mathbf{S}^{\star} \cdot \mathbf{N}\right)_{\times}^{r \times} M \\
& -\left(\dot{\mathbf{N}}+\mathbf{S}^{\star} \cdot \mathbf{N}\right)^{r} \cdot \mathbf{M} \\
& +\operatorname{Tr}\left(\dot{\mathbf{N}}+\mathbf{S}^{\star} \cdot \mathbf{N}\right) \mathbf{M}, \\
& \boldsymbol{\nabla} \times \boldsymbol{\nabla} \psi=2 \dot{\psi} \Omega, \\
& \boldsymbol{\nabla} \cdot(\boldsymbol{\nabla} \times \mathbf{V})=2 \boldsymbol{\Omega} \cdot\left(\dot{\mathbf{V}}+\mathbf{S}^{\star} \cdot \mathbf{V}\right), \\
& \nabla \times(\nabla \mathbf{V})=-\mathbf{E} \times \mathbf{V} \\
& +2 \Omega \cdot\left[\dot{I}-\frac{1}{2} \mathbf{s}^{\star} \times(\mathbf{I} \times \mathbf{V})\right],
\end{aligned}
$$

where $\psi, \mathbf{V}$, and $\boldsymbol{M}$ are, respectively, an arbitrary scalar, vector, and dyadic. The last three space relations clearly look more familiar when $\Omega=0$ so that immersed orthogonal 3-spaces exist.

By appropriately differentiating the curvature equations [(B-14) to (B-17)] and applying the commutation relations to eliminate second derivatives, we arrive at the dyadic form of the 20 Bianchi identities: where $B^{\star}=B-t \times I$. The trace of $(B-26)$ vanishes. The trace of (B-27) is a contracted Bianchi identity:

$$
\boldsymbol{\nabla} \cdot \mathbf{t}+\left[\dot{\rho}+(\operatorname{Tr} S)_{p}\right]=\mathbf{T}: \mathbf{S}-2 \mathbf{a} \cdot \mathbf{t}
$$

The remaining contracted identities are found in a vector equation obtained by subtracting Eq. (B-28) from the antisymmetric part of Eq. (B-26);

$$
\begin{aligned}
& \nabla \cdot T-[\dot{t}+\omega \times \mathbf{t}+(\operatorname{Tr} S)\mathbf{t}]= \\
& \mathbf{t} \cdot \mathbf{S}+\Omega \times \mathbf{t}-\mathbf{a} \cdot \mathbf{T}+\rho \mathbf{a} .
\end{aligned}
$$

It remains only to verify some other relations which are presented in Section II. It is there shown that the quantities defined by

$$
\phi=\frac{d t}{d \tau}=\dot{t}, \quad \mathbf{A}=\nabla t
$$

where $t$ is a timelike coordinate, have a simple interpretation in terms of the space-time metric written in co-moving spatial coordinates:

$$
\phi^{2}=-\frac{1}{g_{010}}, \quad A^{\alpha}=g^{0 \alpha} .
$$

In general, these quantities may always be interpreted as scalar and vector potentials for the acceleration and angular velocity vectors of any timelike congruence. The potential equations given in Section II are, in fact, almost

$$
\begin{aligned}
& \boldsymbol{\nabla} \times \mathbf{Q}-\left(\dot{B^{\star}}+\omega \times \mathbf{B}^{\star}-\mathbf{B}^{\star} \times \omega\right)=-\mathbf{a} \times \mathbf{Q}+\mathbf{P} \times \mathbf{a} \\
& -[\mathbf{S}-(\operatorname{Tr} \mathbf{S}) \mathbf{I}+\Omega \times \mathbf{I}] \cdot \mathbf{B}^{\star} \\
& -\mathrm{S}_{\times}^{\times} \mathrm{B}^{\star T}+\left(\Omega \cdot \mathrm{B}^{\star T}\right) \times 1+2 \Omega \mathrm{t} \text {, } \\
& \boldsymbol{\nabla} \times \mathbf{B}^{\star T}+(\dot{\mathbf{P}}+\boldsymbol{\omega} \times \mathbf{P}-\mathbf{P} \times \boldsymbol{\omega})=-\mathbf{a} \times \mathbf{B}^{\star T}+\mathbf{B}^{\star} \times \mathbf{a} \\
& +[\mathbf{S}-(\operatorname{Tr} \mathbf{S}) \mathbf{I}+\Omega \times \mathbf{I}] \cdot \mathbf{P} \\
& +s_{\times}^{\times} Q-(\Omega \cdot Q) \times I \text {, } \\
& \nabla \cdot P=-(S+3 \Omega \times 1) \dot{x}^{\star},
\end{aligned}
$$


trivial consequences of the commutation relations. Applying Eq. (B-20) to the $t$ coordinate, we have

$$
(\nabla t)^{\bullet}-\nabla(\dot{t})=\mathbf{a} \dot{t}-\mathbf{s}^{\star} \cdot \nabla t
$$

so that using Eq. (B-31)

$$
\phi \mathbf{a}=-\nabla_{\phi}+\dot{\mathbf{A}}+\mathbf{S}^{\star} \cdot \mathbf{A} .
$$

Similarly from Eq. (B-23), we obtain

$$
\nabla \times(\nabla t)=2 i \Omega
$$

or

$$
2 \phi \Omega=\nabla \times \mathbf{A} .
$$

Finally, the time derivative of the natural vectors $\mathrm{e}^{\alpha}=\boldsymbol{\nabla} x^{\alpha}$, as given in Eq. (34) of Section II, is also readily obtained from the commutation relation (B-20), by applying it to the co-moving spatial coordinate $x^{\alpha}$, for which $\dot{x}^{a}=0$. We find

$$
\left(\nabla x^{\alpha}\right)^{\bullet}=-\mathbf{S}^{\star} \cdot \nabla x^{\alpha},
$$

or

$$
\dot{\mathbf{e}}^{\alpha}=-\mathbf{S}^{\star} \cdot \mathbf{e}^{\alpha} .
$$




\section{REFERENCES}

1. Born, M., Ann. Physik, Vol. 30, No. 1, 1909.

2. Herglotz, G., Ann. Physik, Vol. 31, p. 393, 1910.

3. Noether, F., Ann. Physik, Vol. 31, pp. 919-944, 1910.

4. Synge, J. L., Relativity: The General Theory, North-Holland Publishing Co., Amsterdam, Holland, 1960.

5. Rosen, N., Phys. Rev., Vol. 71, p. 54, 1947.

6. Salzman, G., and Taub, A. H., Phys. Rev., Vol. 95, p. 1659, 1954.

7. Rayner, C. B., Compt. Rend., Vol. 248, p. 929, 1959a.

8. Pirani, F. A. E., and Williams, G., Séminaire JANET, 5ième année, Nos. 8 and 9 , 1961 and 1962 .

9. Boyer, R. H., Proc. Roy. Soc. (London), A, Vol. 283, pp. 343-355, 1965.

10. Estabrook, F., and Wahlquist, H., J. Math. Phys., Vol. 5, pp. 1629-1644, 1964.

11. Lichnerowicz, A., Théories Relativistes de la Gravitation et de l'Électromagnétisme, Masson el Cie, Paris, France, 1955.

12. Berenda, C. W., Phys. Rev., Vol. 62, p. 280, 1942.

13. Mфller, C., The Theory of Relativity, Chapter VIII, Oxford University Press, London, England, 1952.

14. Fokker, A. D., Rev. Mod. Phys., Vol. 21, p. $406,1949$.

15. Ehlers, J., and Kundt, W., Gravitation: an introduction to current research, Chapter 2, John Wiley \& Sons, Inc., New York, 1962. 